\title{
Vortex evolution in non-axisymmetric impulsive spin-up from rest
}

\author{
By D. M. HENDERSON${ }^{1}$, J. M. LOPEZ $Z^{1,2}$ \\ AND D. L. STEWAR T ${ }^{1}+$ \\ 'Department of Mathematics, The Pennsylvania State University, University Park, PA 16802, USA \\ ${ }^{2}$ Earth System Science Center, The Pennsylvania State University, University Park, PA 16802, USA
}

(Received 2 July 1995 and in revised form 4 April 1996)

The flow evolution of water in a completely filled rectangular container, impulsively rotated from rest to a steady angular speed, is investigated experimentally and numerically. The pathlines of the fluid from rest to solid-body rotation primarily follow one of two possible configurations that have been described previously in the literature. The first, consisting of two cyclones that form following the separation and roll-up of the sidewall boundary layers and an anticyclone that forms subsequently, results in a pattern on the path to spin-up of cyclonic-anticyclonic-cyclonic vorticity. In the second configuration the cyclones migrate into the interior of the container and merge, resulting in a pattern on the path to spin-up of anticyclonic-cyclonic-anticyclonic vorticity. The experiments provide a parameterization of the possible evolutionary configurations as a function of horizontal and vertical aspect ratios and Reynolds numbers. Critical Reynolds numbers for vortex merger are determined experimentally. Evolutionary configurations in addition to the primary two are observed; in particular symmetry breaking occurs at high Reynolds numbers causing complicated patterns of flow evolution. For some flow conditions at high Reynolds numbers, more than one evolutionary pattern is observed for the same external parameters. The experiments are conducted with a rigid lid showing that a free surface is not required for vortex merger. Numerical integrations of the two-dimensional Navier-Stokes equations (a situation corresponding to the limiting case of a container of infinite depth, where there are no effects from the top and bottom and all flow is horizontal) reproduce qualitatively many of the features of the experimental observations, in particular the merger events. The numerical results show that neither vertical flow due to Ekman boundary layers at the top and bottom nor a free surface are necessary for the observed vortex merger.

\section{Introduction}

The spin-up of nearly inviscid fluids in a container that is initially at rest and then impulsively rotated at a steady angular velocity has been studied in great detail for axisymmetric cylinders (Benton \& Clark 1974; Weidman 1976a,b). In an axisymmetric cylinder, there are two processes contributing to spin-up: viscous diffusion of angular momentum from the sidewall boundary layers and convection of angular momentum due to a vertical pumping of fluid into the Ekman boundary layers on the horizontal surfaces. Viscous diffusion occurs on a time scale $T_{v}=L^{2} / v$, where $v$ is the kinematic

$\dagger$ Present address: ICAM, Virginia Tech University, Blacksburg, VA 24061, USA. 
viscosity of the fluid and $L$ is a horizontal length scale. Ekman pumping of angular momentum occurs much faster, on a time scale $T_{E}=\left(H^{2} / \nu \Omega\right)^{1 / 2}$ where $\Omega$ is the final rotation rate and $H$ is the depth of the fluid. Van Heijst (1989) investigated the spin-up of fluid in non-axisymmetric containers. The inherent lack of azimuthal symmetry introduces an additional mechanism, which can play a dominant role in the spin-up process. The new dynamics are due to the separation of the sidewall boundary layers, which form free shear layers and subsequently roll up into cyclonic cells on a time scale that is fast compared to a rotation period. This is a two-dimensional process; hence, it is independent of $H$. Van Heijst and co-workers (van Heijst 1989; van Heijst, Davies \& Davis 1990; van de Konijnenberg et al. 1994) noted this process in their experiments and Suh (1994) observed it in his numerical study; nevertheless, the nature of the process is not well understood. In particular, there are a number of distinct dynamical states that the system can attain during its evolution from rest to solid-body rotation. The two most common states are a cyclone-anticyclonecyclone $(+-+)$ arrangement, observed experimentally by Stewart (1993) and van de Konijnenberg et al. (1994), and numerically by van de Konijnenberg et al. (1994) and Suh (1994), and an anticyclone-cyclone-anticyclone $(-+-)$ arrangement. The latter arrangement results from the merger of the cyclones. It was observed experimentally by van Heijst et al. (1990) and Stewart (1993). These two patterns (and more) are observed in both the experiments and the two-dimensional computations presented here.

In this paper we present results from a series of experiments in a variety of completely filled rectangular containers with varying horizontal and vertical aspect ratios in order to investigate the processes and parameters that determine vortex evolution during spin-up in rectangular containers. These experiments differ from those of van Heijst et al. (1990) and van de Konijnenberg et al. (1994) in that the flow evolution is investigated by a systematic exploration of parameter space, consisting of Reynolds number and the vertical and horizontal aspect ratios of the container, delineating regions of dynamical behaviour. In the present experiments the fluid completely filled the container so that there was no air between the fluid and the rigid lid, eliminating the effects of free-surface deformations. Van Heijst et al. (1990) observed merger in their free-surface experiments but not in their experiments where a lid was placed on top of the fluid. Van de Konijnenberg et al. (1994) placed a rigid lid on their container, but did not fill it completely, so that they had a deformable free-surface. They did not report any merger events. Stewart (1993) considered the free-surface case only. In addition, we present computations from the two-dimensional Navier-Stokes equations, representing the primary interior flow, which corresponds to the limiting case of a container of infinite depth. These differ from the computations of Suh (1994) in that we compute to higher Reynolds numbers, where merger takes place; Suh (1994) did not report cyclone-cyclone merger events. Further, unlike Suh, we do not enforce the $\pi$-rotational symmetry, which is shown to be broken at high Reynolds numbers in both our experiments and numerics. Van de Konijnenberg et al. (1994) presented three-dimensional computations for low Reynolds numbers where merger does not occur. Van de Konijnenberg (1995) presented three-dimensional computations at higher Reynolds numbers for which merger did occur.

Our main results are the following.

(a) Vortex merger can be characterized by the Reynolds number and aspect ratios. In particular, in experiments with a rigid surface, the cyclone merger occurs at a critical Reynolds number that increases with increasing horizontal aspect ratio (for a fixed vertical aspect ratio). It varies non-monotonically with increasing vertical 
aspect ratio at a fixed horizontal aspect ratio. In particular, it decreases sharply with increasing vertical aspect ratio while the vertical aspect ratio is less than one, then jumps at a ratio of one, and decreases at a lower rate with increasing vertical aspect ratios larger than one. In experiments with a free surface, the critical Reynolds number and the time for merger are less than those measured using the rigid lid.

(b) Vortex merger can occur with either a rigid or free surface as evidenced by the experiments. In the numerical computations, which model the two-dimensional dynamics in the limiting case of infinite depth, merger events also occur.

(c) Ekman pumping is not required for vortex merger as evidenced by the computations, which do not contain the horizontal boundaries.

(d) At high Reynolds numbers, both the experiments and computations show that symmetry breaking can occur, causing a variety of vortex patterns and merger events.

(e) Different flow evolution patterns can result from slightly different start-up conditions.

An outline of the paper is as follows. The experimental apparatus and procedures are discussed in $\$ 2$. The two-dimensional model and the numerical algorithm for its integration are developed in $\$ \$ 3$ and 4 . Symmetries that are inherent in the rectangular geometry and turn out to be either kept or broken in the experimental and numerical results are pointed out in $\$ 5$. Section 6 contains the experimental observations and discusses them in conjunction with the numerical results. Section 7 contains a discussion of the cyclone paths in the various configurations to spin up. Section 8 summarizes the results.

\section{Experimental apparatus}

The experimental equipment consisted of a turntable and drive, a real-time computer, photography systems, a number of rectangular containers, and distilled water with food colouring.

The rotating table is a Lintech model MR-308180 that rotates about its centre to within $0.05 \mathrm{~mm}$. The rectangular container is centred on the table with a pin to within $0.05 \mathrm{~mm}$. The table is level to within $5.8 \times 10^{-6} \mathrm{rad}$. When the drive is powered, the table exhibits high-frequency vibrations that vary in frequency and intensity with the rotation rate. Stewart (1993) documented the spectra of the free-surface response to these vibrations. However, unless otherwise noted, all of the experiments discussed herein were conducted in containers with rigid lids, rather than free surfaces. The rotating table assembly was mounted on a structural wall of the laboratory to isolate it from extraneous laboratory vibrations.

The computer-generated command signal to the motor drive was varied impulsively (a time interval of $10^{-4} \mathrm{~s}$ ) from a zero voltage to the final voltage in the majority of the experiments. To determine if the flow patterns depended on the initial condition, experiments were also conducted in which the command signal was ramped linearly from zero to a final voltage in a specified time interval. In the discussions of the results, all of the experiments had an impulsive start unless otherwise noted. The table drive was a DigiPlan Electronic Motion Control brushless d.c. motor with velocity feedback. Because the drive had velocity feedback instead of position feedback, it had to be checked before each experiment to ensure that a zero voltage from the computer provided zero motion. The angular speed of the table was measured with an accuracy of $\pm 0.005 \mathrm{rad} \mathrm{s}^{-1}$ using either a tachometer or a stopwatch during each experiment. The servosystem operates for table speeds less than about $1.8 \mathrm{rad} \mathrm{s}^{-1}$ providing an 


$\begin{array}{cccc}\text { Container } & L(\mathrm{~cm}) & W(\mathrm{~cm}) & H(\mathrm{~cm}) \\ 1 & 12.7 & \text { variable } & 10.0 \\ 2 & 10.5 & 23.9 & 14.7 \\ 3 & 16.4 & 37.4 & \text { variable } \\ 4 & 15.3 & 34.9 & \text { variable } \\ 5 & 15.3 & 34.9 & 10.0 \\ 6 & 10.5 & 23.9 & 10.0 \\ 7 & 11.0 & 25.1 & \text { variable }\end{array}$

TABLE 1. Dimensions of containers used in results shown in figures 9 and 12

upper bound on the angular speeds attainable. In all of the experiments herein, the table was rotated in the counter-clockwise direction.

The experiments used a number of containers with rectangular cross-sections; the largest horizontal and vertical lengths were about $30 \mathrm{~cm}$ and $60 \mathrm{~cm}$. The containers were constructed of lucite plates that were joined with methylene chloride. The horizontal bottom was machined to be flat; the sidewalls were not. After gluing, the long walls exhibited some warping so that the short dimension, $L$, varied by almost $1 \%$ across the width of the container. This variation in $L$ is of the same order as the boundary layer thickness. Two of the containers were equipped with inserts that provided removable endwalls and spacers, so that the horizontal aspect ratio could be varied from 3 to 2 . Three containers were equipped with spacers and a false bottom, so that the vertical aspect ratio could be varied from 0.20 to 5.5 . The containers were fastened to the table to ensure that they did not slip during start-up and were filled with distilled water. Bubbles on the wetted surfaces were removed with a paint brush. In the rigid-lid experiments, the containers were slightly overfilled. A clear lucite lid was slid across the surface ensuring that no bubbles formed in the fluid domain. The lid was then screwed down to ensure that it did not slip during the impulsive start-up. The dimensions of the containers used in the experiments shown in figures 9 and 12 are given in table 1.

To visualize the flow, red and blue food colouring was dropped into the leading corners (and in some cases in the centre) of the container before setting the lid on. The food colouring was applied at the surface and sank, creating a column through the water and a blob on the bottom. Still photographs and videos were obtained with cameras mounted on a frame attached to the rotating table.

Many of the experimental results presented catalogue the flow patterns observed during spin-up in terms of the Reynolds number and horizontal and vertical aspect ratios for each experiment. These parameters depend on measurements of the length scales of the containers, the viscosity of the water, and the angular rotation rate of the container. The water temperature was not monitored during the experiments whose results are shown in figure 9. For the experiments whose results are shown in figure 12, water from the same source as that used for the experiment was poured into a beaker at the same time as water was poured into the experimental container. The temperature of the water in the beaker was monitored, and the value of viscosity used in calculating Reynolds numbers for figure 12 corresponded to the tabulated value for that temperature from Weast (1976, p. F-51). However, it was later observed that the temperature of the water in the container may have been as much as $1{ }^{\circ} \mathrm{C}$ higher than the water in the beaker. (The temperature of the water for experiments conducted in container 7 was measured in the container before each experiment.) For the 
results in figure 9 , a viscosity of $v=0.0100 \mathrm{~cm}^{2} \mathrm{~s}^{-1}$ was used in determining Reynolds number. The actual temperature may have varied, as a conservative estimate, between 17 and $22{ }^{\circ} \mathrm{C}$ over the months during which the experiments were conducted. The corresponding change in fluid viscosity is $0.009548 \leqslant v \leqslant 0.01081 \mathrm{~cm}^{2} \mathrm{~s}^{-1}$. This range of viscosity in addition to the measurement accuracy of lengths and rotation speeds represents as much as an $8 \%$ ambiguity in the value of the Reynolds numbers depicted in figure 9, as a conservative estimate. The error in Reynolds number in figure 12 is as much as $3 \%$. The viscosity used for parameters quoted in figures 2,5 , $8,10,11$, and 13 also used $v=0.0100 \mathrm{~cm}^{2} \mathrm{~s}^{-1}$.

Hard copies of the videos of the flow evolution were used to locate cyclone centres as a function of time. This procedure was somewhat subjective, since it is difficult to determine the centre of the cyclones from the snap-shots of the dye-lines. While the dye-lines of the cyclones remain circular, the centre is reasonably well identified, but as they become more distorted during interaction and merger, the dye at the centre becomes elongated. Thus the centre was identified as the centre of the elongated region. Section 7 presents a comparison of the tracks of the centres obtained from the experiments with those obtained from computations of streamlines. The dyelines and the instantaneous streamlines do not coincide, especially during the rapid development stage associated with merger. Further, the centres of the instantaneous streamlines do not necessarily coincide with vorticity maxima, which in turn are not always a good indicator of a vortex centre (Jeong \& Hussain 1995).

Spin-up times were measured for low-Reynolds-number flows $\left(\operatorname{Re} \sim O\left(10^{3}\right)\right)$ by viewing videos of the evolving dye-lines taken in the rotating frame. By viewing the videos at high-speed, the velocity gradients were large enough so that a time was observable when the dye-lines seemed no longer to be evolving. These rather subjective measurements are not a quantitative measure of velocities, but provided a reproducible time by which the fluid was approximately in solid-body rotation.

\section{Formulation of the primary flow problem}

From experimental observations and classical approaches, the flow field away from boundaries can be decomposed into a primary flow and a secondary flow. The primary flow's vortex lines are parallel to the rotation axis; hence, its velocity field is everywhere perpendicular to its vorticity field. So, in a coordinate system with one coordinate direction, $z$, aligned with the rotation axis, the primary flow is in general planar two-dimensional. If it also has azimuthal symmetry, then the primary flow is one-dimensional, varying spatially only with radial distance from the axis. The secondary flow is induced by the bending of the primary flow's vortex lines, such as by the centrifugal instability responsible for the onset of Taylor vortex flow (a secondary flow) from circular Couette (the primary flow) in the ideal Taylor-Couette problem. The vortex line bending can also result from kinematic constraints, such as the presence of rigid surfaces not parallel to the rotation axis. In the case under investigation here, these rigid surfaces are the top and bottom of the container.

For the nonlinear, non-axisymmetric situation considered here, we investigate the two-dimensional dynamics of the primary flow alone, without the influence of Ekman pumping or the advection and stretching of the vertical vorticity by the secondary flow. This model provides some indication of the extent to which the experiments are affected by Ekman pumping. This two-dimensional model, together with the experiments using a rigid lid, permits the isolation of the various influences on the system. Specifically, the free surface deformation is eliminated, both in the 
experiments and the model. Further, the model eliminates the effects of Ekman pumping, thus allowing an examination of the dynamics due solely to horizontal viscous diffusion, two-dimensional boundary layer separation, and two-dimensional advection of vorticity.

The governing equations with time scaled by $1 / \Omega$ and length scaled by $L$, the short horizontal length of the rectangular container, reduce to the evolution equation for the vertical component of vorticity, $\zeta$, in a frame of reference rotating at angular velocity $\Omega$ :

$$
\zeta_{t}+\psi_{y} \zeta_{x}-\psi_{x} \zeta_{y}=\nabla^{2} \zeta / R e,
$$

where the stream function $\psi(x, y, t)$ is determined kinematically from

$$
\begin{aligned}
& \nabla^{2} \psi=-\zeta ; \\
& \operatorname{Re}=\Omega L^{2} / v
\end{aligned}
$$

is the Reynolds number, and $v$ is the kinematic viscosity. The boundary conditions are that the flows tangential and normal to the boundaries vanish, i.e. $\psi$ is a constant, say 0 , on the sidewalls of the rectangular container. The fact that there is no tangential flow on the sidewalls together with (3.2) yields $\zeta=-\psi_{n n}$ on the sidewalls, where the subscripts denote differentiation normal to the wall. Then

$$
\zeta\left(-\delta_{h} / 2, y\right)=\zeta\left(\delta_{h} / 2, y\right)=-\psi_{x x},
$$

and

where

$$
\zeta(x,-1 / 2)=\zeta(x, 1 / 2)=-\psi_{y y},
$$

$$
\delta_{h}=W / L
$$

is the horizontal aspect ratio of the container and $W$ is its long horizontal length. This two-dimensional system corresponds to the limiting case where the vertical aspect ratio of the container,

$$
\delta_{v}=H / L,
$$

goes to infinity. For the case of impulsive spin-up from rest, the initial condition is $\zeta=-2 ;$ the Rossby number $R o=\Delta \Omega / \Omega=1$, and the Taylor number $T a=\Omega L^{2} / v$. In general, $R e=R o T a$. The Rossby number is the ratio of convective to Coriolis accelerations and gives an estimate of the relative importance of the nonlinear terms. The Taylor number is the ratio of Coriolis force to the viscous force in the fluid. The Reynolds number is the ratio of convective to viscous force. So, for nonlinear impulsive spin-up from rest, the primary flow is governed by only one dynamic nondimensional parameter $R e=T a$, and by a geometric parameter, the horizontal aspect ratio $\delta_{h}$.

\section{Numerical method of solution for the primary flow}

The system of equations (3.1) and (3.2) is solved using second-order central differences on a non-uniform grid designed to resolve the thin sidewall boundary layers formed following the impulsive start. The stretched grid is given by

$$
x_{i}^{\star}=\delta_{h}\left(x_{i}+a \sin \left(2 \pi x_{i}\right)\right)
$$

and

$$
y_{j}^{\star}=y_{j}+b \sin \left(2 \pi y_{j}\right),
$$


where $x_{i}=-1 / 2+(i-1) /(n x-1), i=1$ to $n x$, and $y_{j}=-1 / 2+(j-1) /(n y-1)$, $j=1$ to $n y$. In all the results presented, the stretching factors used are $a=b=0.1$. This choice concentrates points near the boundaries, but not to the extent where the interior resolution suffers. The ratio of maximum to minimum grid spacing over the whole domain is approximately 4.4. Time integration of (3.1) is accomplished by a second-order predictor-corrector scheme.

The discrete version of (3.2) is solved for $w$ on the interior grid points using the generalized cyclic reduction method of Sweet (1974). The values of $\zeta$ on the sidewalls are evaluated by discretizing derivatives with one-sided differences, and by using (3.2) and the boundary conditions on $y$.

Extensive tests have been run to ensure that the solutions presented are independent of spatial and temporal resolution for $R e=10^{4}$ and $\delta_{h}=2.28$ as a test case. Typically, the errors in $\psi$, are an order of magnitude smaller than the errors in $\zeta$. It was found that $n y \geqslant 301$ was needed to sufficiently resolve the vorticity field. The temporal discretization was indeed found to be second-order accurate. Using approximately the largest $\delta t=1.25 \times 10^{-3}$ for which the scheme is stable on the $301 \times 685$ grid results in about 6-figure accuracy. All the computations presented here were performed using 64-bit arithmetic on an IBM RS6000 SP2.

\section{Symmetries of the system}

Although the distinguishing feature between spin-up in the rectangular container presented here and the classical spin-up in an axisymmetric right-circular container is the lack of azimuthal symmetry, the present system also has a large degree of symmetry. Owing to the presence of the rigid top endwall, the system has reflectional symmetry about the container half-height at $z=H / 2$. This reflection plane may be thought of as a non-deformeable free surface. There are also symmetries in the horizontal direction. The system is invariant to rotations of $\pi$ rad centred about the rotation axis (in a circular cylinder, the system is invariant to any rotation and in a square container, it is invariant to rotations of $\pi / 2 \mathrm{rad}$ ). For low $R e$, we expect the flow to preserve these symmetries and for increasing $R e$ perhaps to break them. The experiments suggest that horizontal symmetry breaking first occurs for $R e$ near that required for the merger of the cyclonic cells. Whilst we observe this correlation, we have no basis to claim any causal relationship. The numerics show non-trivial departures from the horizontal symmetry for $R e>10^{4}$.

A further observation regarding the symmetry of this system is that there exist two distinct states related by a reflection. If $\left(\zeta_{1}(x, y, t), \psi_{1}(x, y, t)\right)$ is a state satisfying (3.1) and $(3.2)$, then so is $\left(\zeta_{2}(x, y, t), \psi_{2}(x, y, t)\right)$ where

$$
\zeta_{2}(x, y, t)=-\zeta_{1}(-x, y, t)=-\zeta_{1}(x,-y, t),
$$

and

$$
\left.\left.\psi_{2}(x, y, t)=-\psi_{1}(-x, y, t)\right)=-\psi_{1}(x,-y, t)\right) .
$$

These alternative states are arrived at trivially mathematically. In some experiments for which the external parameters were the same, we did observe two flow states that appeared to be related by the reflectional symmetry (5.1) and (5.2). We will re-address the symmetries of this system and describe the experiments with the multiple states in $\$ \$ 6.4$ and 6.5 . 


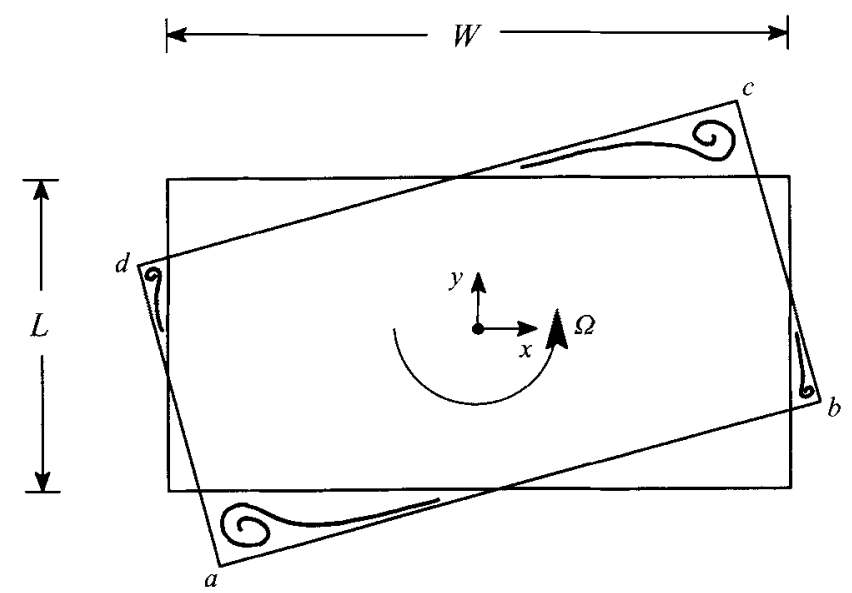

FIGURE 1. Schematic plan view of the rectangular container. The corners $a$ and $c$ are termed leading corners and $b$ and $d$ are trailing corners.

\section{Results from laboratory and numerical experiments}

Herein we describe the flow evolution from rest to solid-body rotation (in the inertial reference frame) observed in the experiments. In particular, we show photographs of the pathlines of the flow as a function of various parameters of the problem including the horizontal aspect ratio $\delta_{h}=W / L$, the vertical aspect ratio $\delta_{v}=H / L$, and the Reynolds number $R e=\Omega L^{2} / v$. Figure 1 shows a schematic plan view of the container at rest and displaced a small amount after start-up with the 'leading' corners, $a$ and $c$, and the 'trailing' corners, $b$ and $d$ labelled for discussion. Upon viewing the photographs, recall that they are of the pathlines of the flow, not the streamlines. Thus, the 'vortices' in the photographs may or may not indicate vorticity. For example, many of the photographs shown are taken after the fluid was essentially spun up, when the velocities approached zero (in the rotating frame); thus, the circular pathlines show the history of vortices, rather than active vortices.

Results from numerical computations are also discussed. We do not attempt to make a direct quantitative comparison between experimental and numerical results; rather, we seek to use both in a complementary fashion to help understand the flow.

\subsection{Cyclone formation and evolution}

The experiments demonstrate two main types of flow evolution. For lower Reynolds numbers, $O\left(10^{3}\right)$, a three-cell pattern of cyclonic-anticyclonic-cyclonic $(+-+)$ vorticity developed. For higher Reynolds numbers the two cyclonic cells merged at the centre resulting in a three-cell pattern of anticyclonic-cyclonic-anticyclonic $(-+-)$ vorticity.

The evolution of the flow at low Re was described by Stewart (1993) and van de Konijnenberg et al. (1994); an example with a rigid top is shown in figure $2 a$. In these overhead photographs of the pathline evolution, drops of blue, red, and yellow dye were initially introduced in corners $a, c$, and the centre respectively. Upon onset of rotation, boundary layers develop on the sidewall, top, and bottom boundaries. The Ekman layers on the top and bottom boundaries cause fluid there to be accelerated outward into the sidewall layers. A slow flow of dye from the bottom Ekman layer was observed to wind its way up the sidewall boundary layer (there is presumably a corresponding flow from the top Ekman layer, but this is not visualized as the dye 
settles primarily in the bottom portions of the container). Immediately following the impulsive start, the sidewall layers begin to separate, forming free shear layers that roll up near the corners to form cyclonic cells (e.g. $t=3$ in figure $2 a$ ). These cyclonic cells form in all four corners. They are not identical except in the degenerate case of $\delta_{h}=1$. As in the schematic of figure 1 , the cyclones that form in the leading corners are larger and eventually dominate those formed in the trailing corners. The cyclonic cells in the leading corners continue to grow until their diameter is comparable to $L$. Their final size and location depends primarily on $\delta_{h}$ as will be discussed below. A view from the side of the container shows that the cyclones wind up and outward during the early part of the evolution. After a few rotation periods they form concentric columns that appear quite uniform in the vertical; the direction of the weak vertical flow cannot be determined from the dye-lines after a few rotation periods. The secondary, vertical flow is quite weak (of $O\left(10^{-3} \zeta\right)$ according to Pedlosky 1984), and cannot be resolved with the flow visualization used here once the dye is distributed vertically.

Observations in the rotating reference frame show that the cyclones initially have an angular velocity that is larger than that of the rotating container. This is also borne out in the computations. Even for $R e=2.4 \times 10^{3}$, the computations show that by $t=10$ the peak relative vorticity of the cyclones is $>2$ and for $t>15$, their vorticity decays, as indicated by the profiles shown in figure 3 of $\zeta$ through the midplane $y=0$ at various times as indicated. For larger $R e$, the cyclone's $\zeta$ is even larger. For example, figure 4 shows $\zeta$ through the midplane for $R e=10^{4}$ and $\delta_{h}=2.2$. At $t=18$, the peak $\zeta$ in this plane is $\approx 11$ (the peak $\zeta$ of the cyclones is larger as the peaks do not reside in the plane at this time), and even after the merger, at $t \approx 70$, the peak $\zeta$ of the resultant cyclone in the centre is $\approx 5.5$. Note also that for $R e=10^{4}$, the peak negative $\zeta$ is $<-2$, getting as large as -4 at times. These peak negative $\zeta$ at large $R e$ are a result of sidewall boundary layer separations rather than anticyclones being spun-up by shear from surrounding cyclones, as is the case with the anticyclone in the centre of the $R e=2.4 \times 10^{3}$ flow. Only in the sidewall layers of the $R e=2.4 \times 10^{3}$ flow is $\zeta<-2$. In summary, the spin-up of the fluid in the rectangular container consists largely of a spin-down of the cyclones. For low-Re flows the cyclones transfer angular momentum to the fluid in the centre of the container by viscous shear. The fluid in the centre of the container initially has zero velocity (in the inertial reference frame) and remains essentially stationary until $t \approx 10$ when it begins to spiral due to shear from the cyclonic cells.

\subsection{Low-Re flows}

There appear to be two dominant time scales for low-Re flows. The first is the time for the cyclones to form, $O(1 / \Omega)$ (i.e. the cyclones form within one rotation period regardless of the size, speed, or depth of the container). The second is the time for the cyclones to transfer their angular momentum to the anticyclone until the whole system is in solid-body rotation. Figure 5 shows measurements of the time, $\tau$, for spin-up to solid body rotation for two sets of experiments in which there was no merger: one in which the depth was varied and one in which the rotation rate was varied. Note that unlike the rest of the experiments discussed herein, the experiments depicted in figure 5 used a free surface, rather than a rigid lid. Figure 5 shows that $\tau / \Omega$ scales like the Ekman time scale, $T_{E}=\left(H^{2} / v \Omega\right)^{1 / 2}$. In contrast to the axisymmetric case where the velocities scale with $T_{E}$ throughout the spin-up process, here the scale applies only after the cyclones have essentially reached their final locations. The data in figure 5 support the assertion of van Heijst et al. (1990) that the time scale for the decay of the cells is the Ekman time scale. 
(a) $\operatorname{Re}=2360$

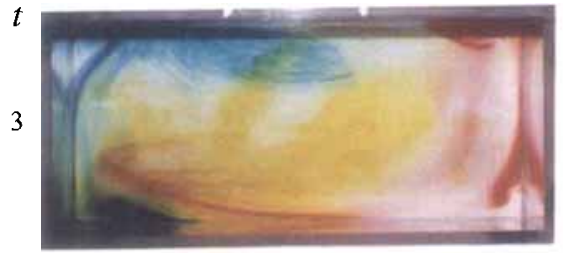

5
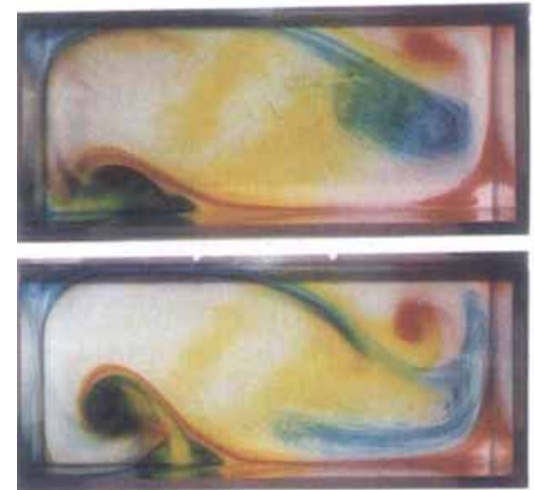

9

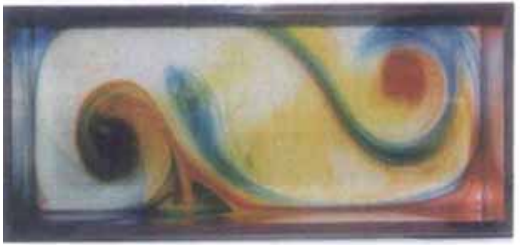

10

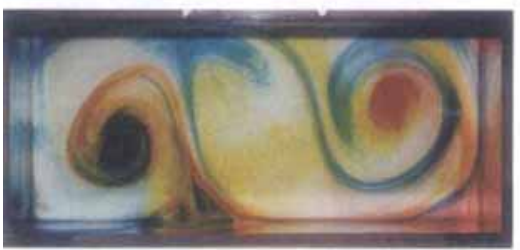

14

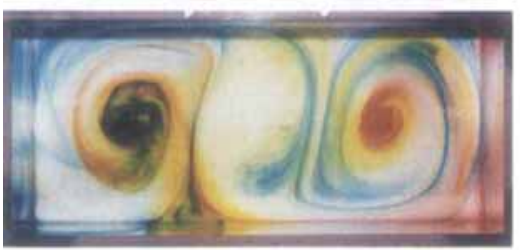

40

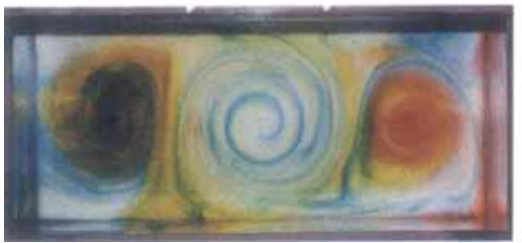

(b) $\operatorname{Re}=12900$

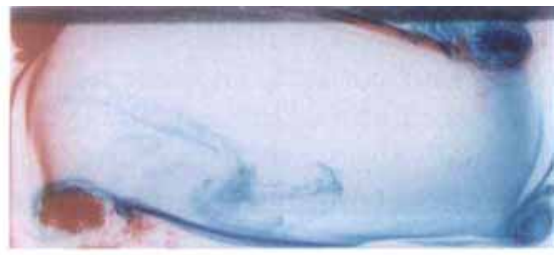

3.8

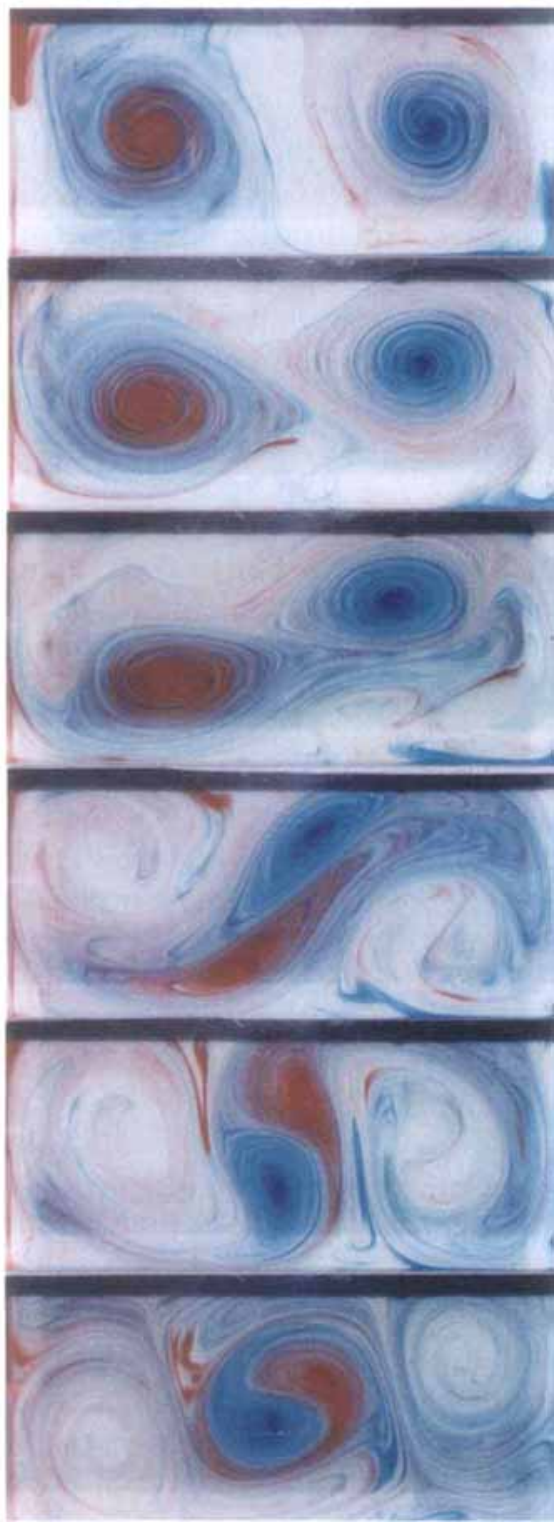

17.8

24.8

31.6

39.0

46.0

95.3

Figure 2. Overhead photographs of the evolution of dye-lines in (a) a non-merger experiment at various non-dimensional times $t$ with $\delta_{h}=2.28, \delta_{v}=0.65, R e=2360$, and $L=15.3 \mathrm{~cm}$; and $(b)$ a merger experiment at various non-dimensional times $t$ with $\delta_{h}=2.20, \delta_{v}=0.79, R e=1.29 \times 10^{4}$, and $L=12.7 \mathrm{~cm}$. 


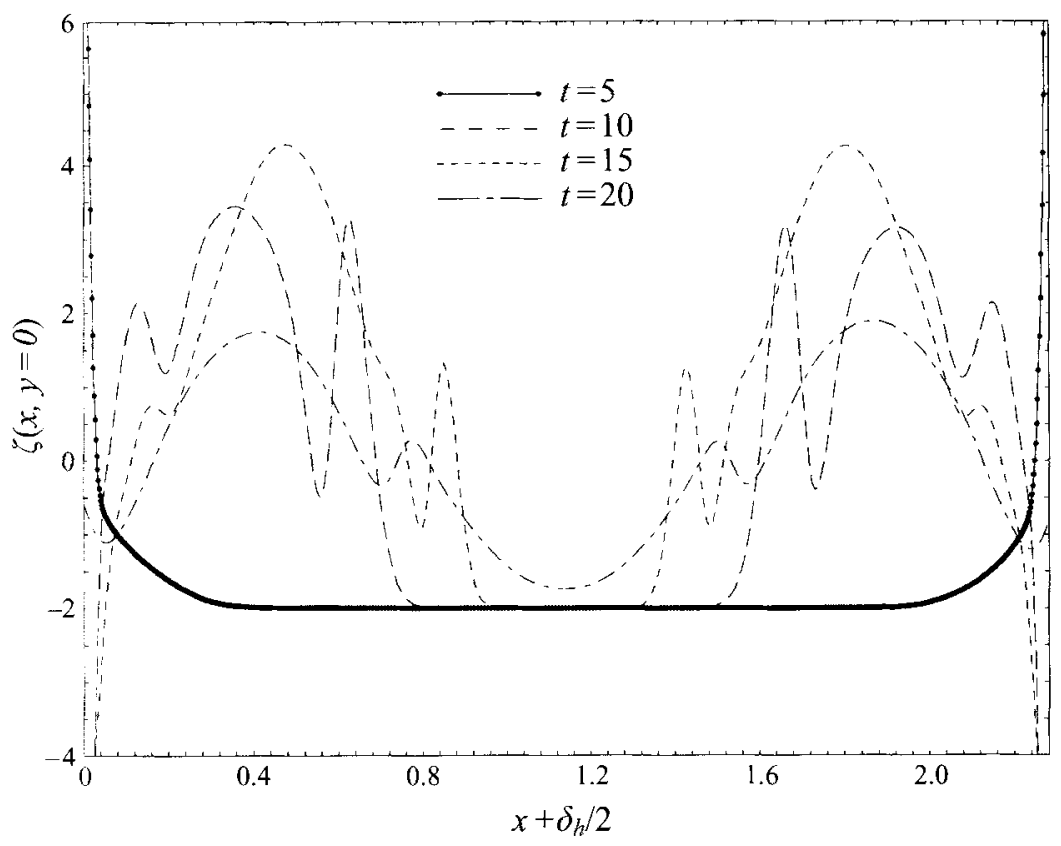

FIGURE 3. $\zeta$ on the midplane $y=0$ for $R e=2.4 \times 10^{3}, \delta_{h}=2.28$ at times as indicated from the calculation on the $(301 \times 685)$ grid with $\delta t=1.25 \times 10^{-3}$.

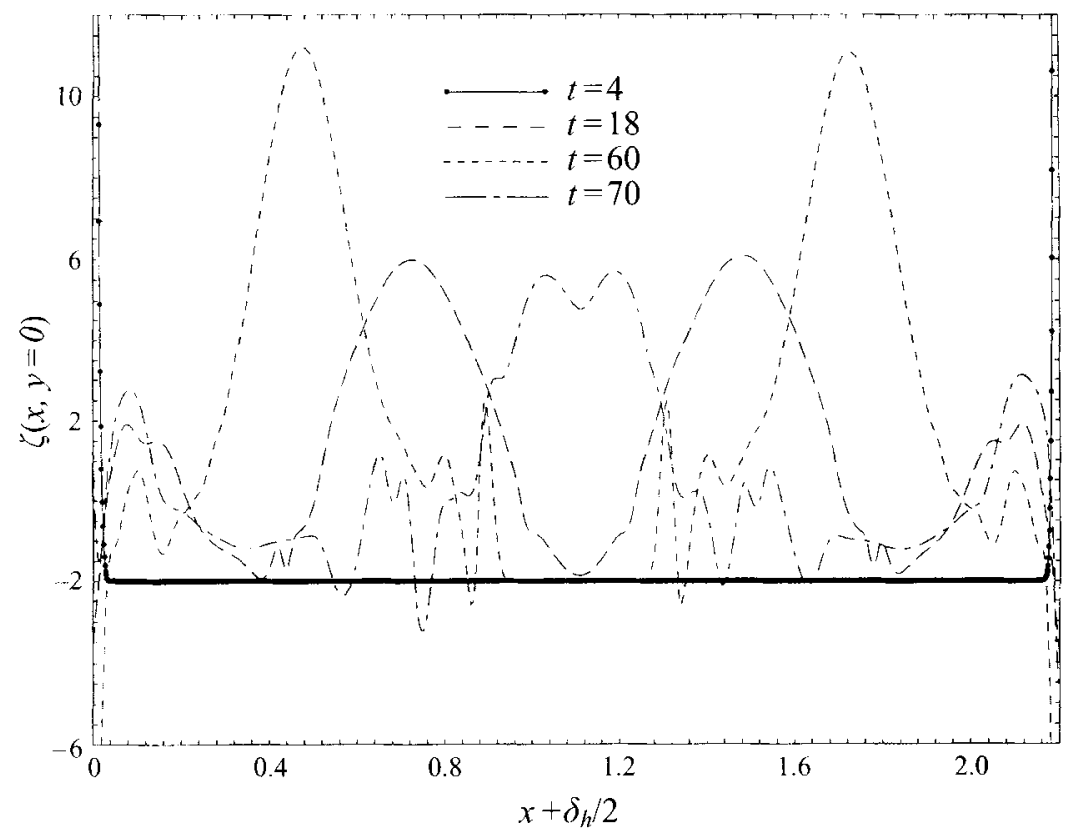

FIGURE $4 . \zeta$ on the midplane $y=0$ for $R e=10^{4}, \delta_{h}=2.20$ at times as indicated from the calculation on the $(301 \times 661)$ grid with $\delta t=1.25 \times 10^{-3}$. 


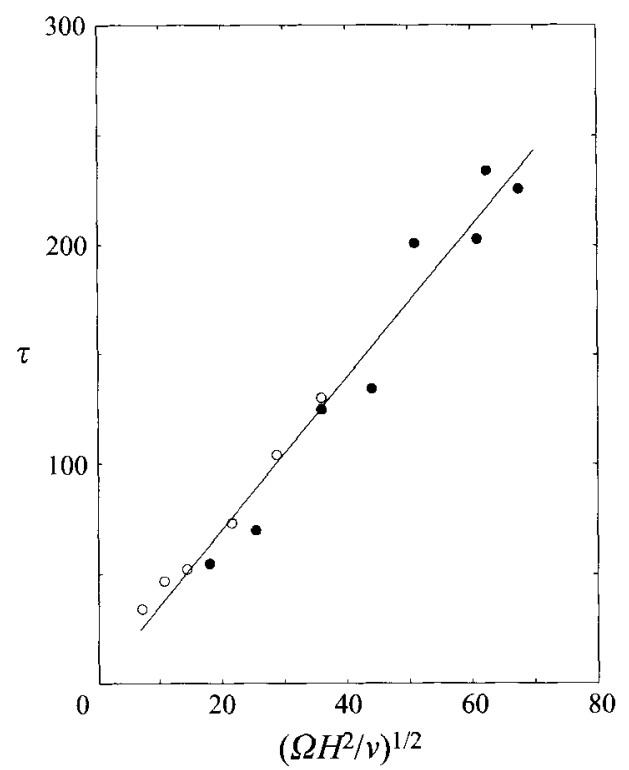

Figure 5. Measured times for spin-up (non-dimensionalized by the rotation rate) as a function of $T_{E} \Omega$ for two sets of experiments, one in which the depth was fixed and the rotation rate was varied $(\bullet)$, and one in which the rotation rate was fixed and the depth was varied (o), both for $\delta_{h}=3.0$. The line shows a least-squares fit such that $\tau=3.47\left(H^{2} \Omega / v\right)^{1 / 2}+0.31$.

Stewart (1993) presented photographs of the pathlines to spin-up for varying depths with a fixed $\Omega$, and for varying $\Omega$ with a fixed depth, both for a given horizontal aspect ratio. Those experiments have shown that if the depth is 'shallow enough', or if $\Omega$ is 'large enough', then the system spins up before the cyclones have a chance to move significantly out of the corners from where they are initiated. The data in figure 5 and the corresponding photographs from Stewart (1993) show that when $T_{E}$ is less than approximately $10 / \Omega$, the cyclones do not have sufficient time before spin-up to move significantly out of the corners. Thus, in experiments for which $T_{E}$ is less than approximately $10 / \Omega$, Ekman pumping due to the horizontal boundaries dominates in the spin-up process. However, in experiments for which the Ekman time scale is large compared to the rotation period, the dominant dynamics as manifested in the cyclone evolution, is primarily due to the effects of the sidewalls. In such experiments, which are the focus of the present study, the fact that the evolution of the anticyclones in the centre of the container is much slower than the evolution of the cyclones, and correspondingly, the fact that the Ekman time scales are long compared to the rotation rates, are qualitative indications that Ekman pumping is a weak process in the overall evolution of the system.

The slower spin-up time for anticyclones is also reflected in the computations of the dynamics of the interior primary flow (in which there is no Ekman pumping) depicted in figure 6 , which shows $\zeta$ and $\psi$ for $R e=2.36 \times 10^{3}$ and $\delta_{h}=2.28$ for the various times during the spin-up that correspond to the photographs in figure $2(a)$. We emphasize again that the photographs are of pathlines, rather than vorticity or streamlines. Nevertheless, there is a striking correspondence between the experiment and the numerical simulation when comparing the sizes of the cells and their locations at various times. This comparison does not involve flow velocities, which were not measured here. Hence, figures $2(a)$ and 6 show that the two-dimensional dynamics 
of the primary interior flow are adequate in describing the flow patterns and their corresponding times when the Reynolds numbers are small. They say nothing about the two-dimensional model's capability of predicting flow velocities. Note that in both the experiments in figure $2(a)$ and the computations in figure 6 there is evidence of secondary boundary layer separation behind the cyclone that is forming in corner $a$ at about $t=5$. We note that at this low $R e$, the stability of the numerics is dominated by the diffusion requirement (Williams 1967), $\delta t<\delta x^{2} R e / 8$. For stable computations, a $\delta t$ of half that used for $R e=5 \times 10^{3}$ was needed for the $R e=2.36 \times 10^{3}$ computation on the same spatial grid, suggesting that viscous effects are important in the horizontal dynamics of flows with $R e$ in this range.

\subsection{High-Re flows}

The evolution of the flow at high Reynolds numbers was first described by van Heijst et al. (1990) and later observed by Stewart (1993). The initial formation of the cyclonic cells in the corners is as described above for the low-Re flows. However, for high $R e$ the cyclones continue to evolve past the state shown in figure $2(a)$; they travel towards the centre of the container, rotate around each other, and merge, as shown in figure $2(b)$. Here again, figure $2(b)$ shows overhead photographs of the evolution of the pathlines in a particular experiment. We have shown photographs of the merger process for a flow with $\delta_{v}=0.79$, because in deeper containers for which the merger process looks essentially the same, the photographs are less vivid because the dye is spread over a large column. The videos of the merger and non-merger experiments show that as in the numerical computations shown in figures 3 and 4 , the cyclones in the merger experiments are initially more intense than those in the non-merger experiments. From these videos, for $R e \sim O\left(10^{4}\right)$, we estimate that the cyclones rotate about three to four times faster than the container.

Figure 7 shows computations of $\zeta$ and $\psi$ for $\delta_{h}=2.2$, and $R e=10^{4}$. The Reynolds number is less than that of figure $2(b)$; in the computations when $R e=1.29 \times 10^{4}$, the flow exhibited symmetry breaking (see figure 10), rather than a clean merger event like those depicted in figures $2(b)$ and 7 . The point of this qualitative comparison is to show that a merger event of the same type as observed in the experiments can be captured by the two-dimensional model of the interior primary flow. Thus, the weak secondary flow, contributing vertical motions, is not a prerequisite for vortex merger in this system. However, we note that the merger process and vortex evolution in the experiments occurs much faster than in the numerics as shown by the times listed in figures $2(b)$ and 7 . The differing time scales of the high-Re evolution between the experiments and the computations of the interior primary flow are likely to be due to Ekman pumping in the experiments. The experiments correspond to $\delta_{v}=0.79$, whereas the computations correspond to the limit $\delta_{v} \rightarrow \infty$. Figure 5 shows that $\tau \propto H$ as is $T_{E}$. In the experiments, $H$ is finite, so that Ekman pumping, though a secondary effect, provides an additional spin-up mechanism that is not present in the numerics.

Experiments also indicate that the time for merger depends on $\delta_{h}$. For $\delta_{h}<2.5$ the time for the merger process to begin is about $25 \pm 5$ time units, independent of the Reynolds number, while for $\delta_{h}>2.5$ it varied between about 50 and 80 time units, depending on the Reynolds number. In the computations, with $\delta_{h} \approx 2.2$ and $\delta_{v} \rightarrow \infty$, for the Re values where the cyclones merge, they do so after about 60 time units.

\subsection{Symmetries observed in the experiments}

The final flow patterns of the non-merger and merger cases demonstrate a horizontal symmetry, as discussed in $\$ 5$. Figure 2(a) $(t=40)$ shows that in the non-merger case, 
(a)
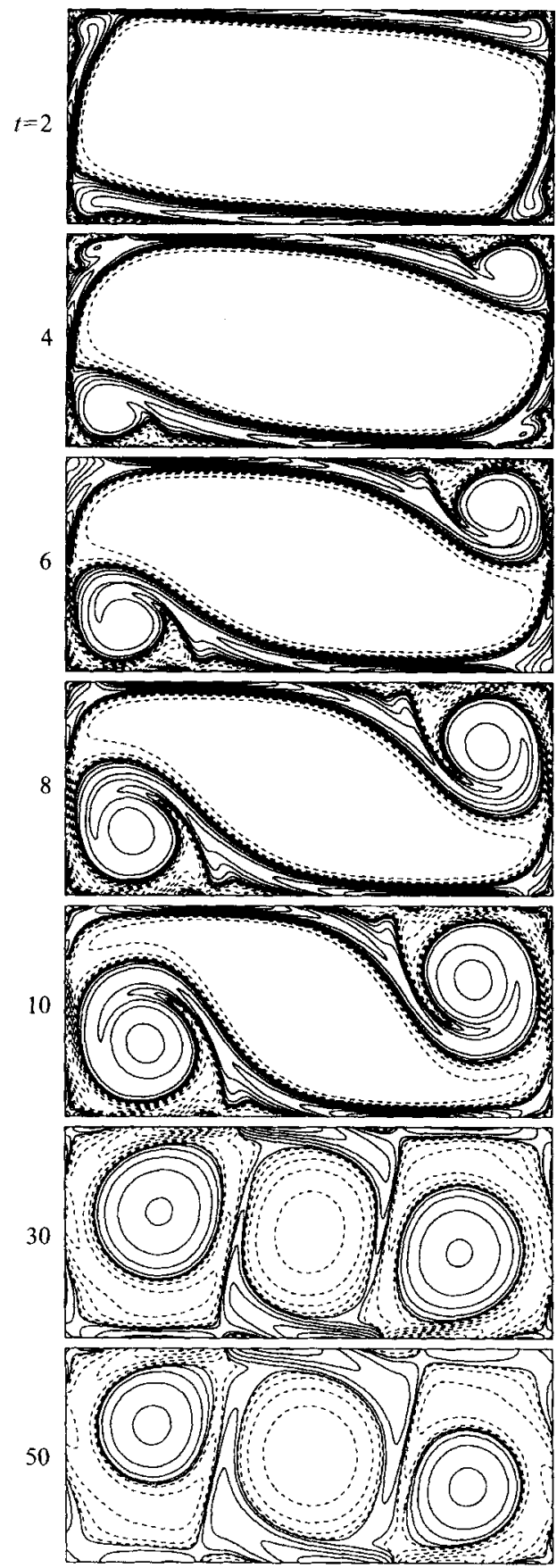

(b)
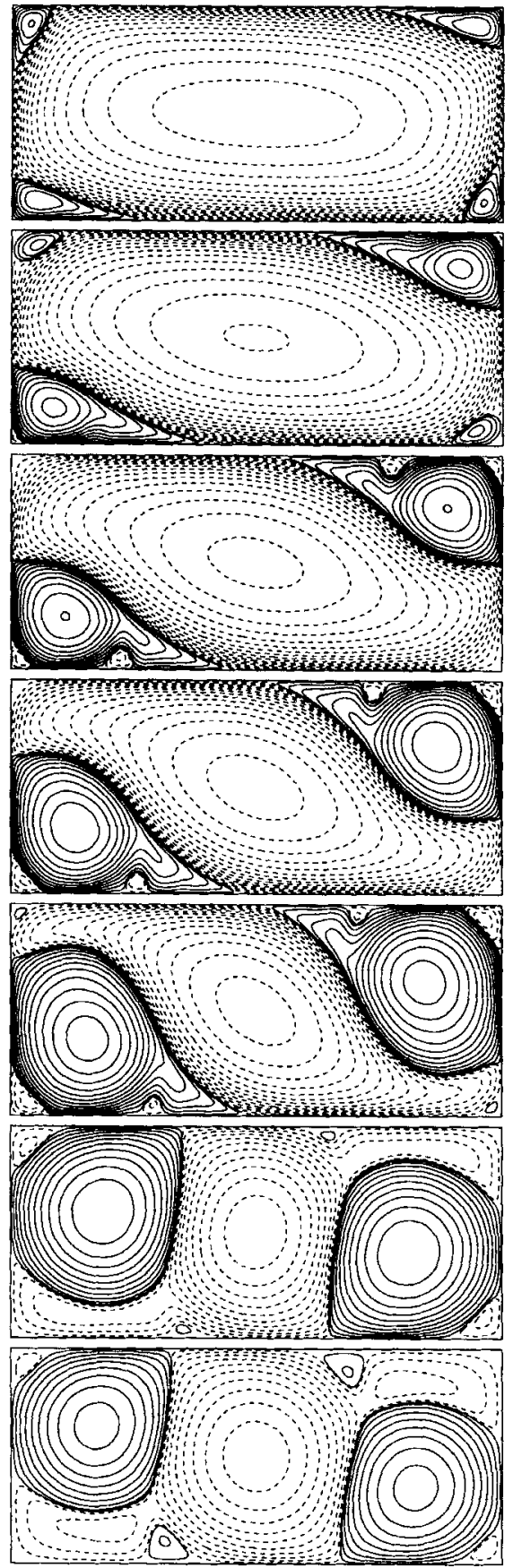

FIGURE 6. Contours of $\zeta(a)$ and $\psi(b)$ at the times as indicated for $R e=2.36 \times 10^{3}$ and $\delta_{h}=2.28$ calculated a grid of size $151 \times 343$ with $\delta t=1.25 \times 10^{-3}$. The contour levels are non-uniformly spaced, with 20 positive (solid lines) and 20 negative (dashed lines), determined by c-level $(i)=\operatorname{Max}($ variable $) \times(i / 20)^{3}$ and c-level $(i)=\operatorname{Min}($ variable $) \times(i / 20)^{3}$ respectively, with $i=1$ to 20 . For all the plots, the following have been used: $\operatorname{Max}(\zeta)=-\operatorname{Min}(\zeta)=100$ and $\operatorname{Max}(\psi)=-\operatorname{Min}(\psi)=0.3$. 
(a)

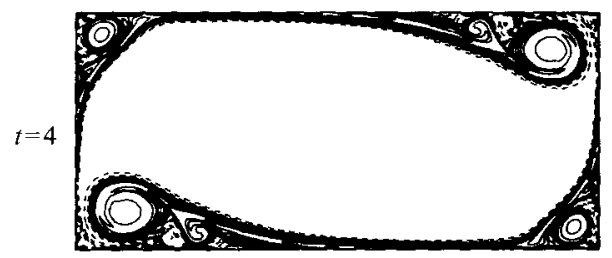

18

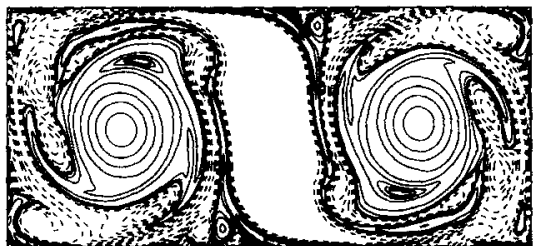

60

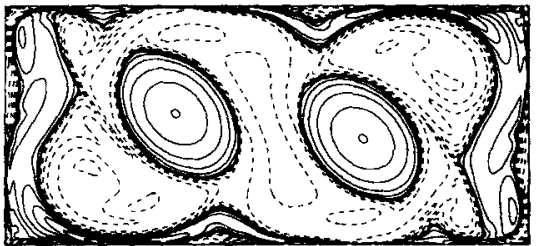

62

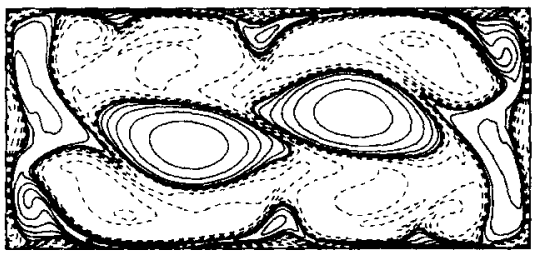

64
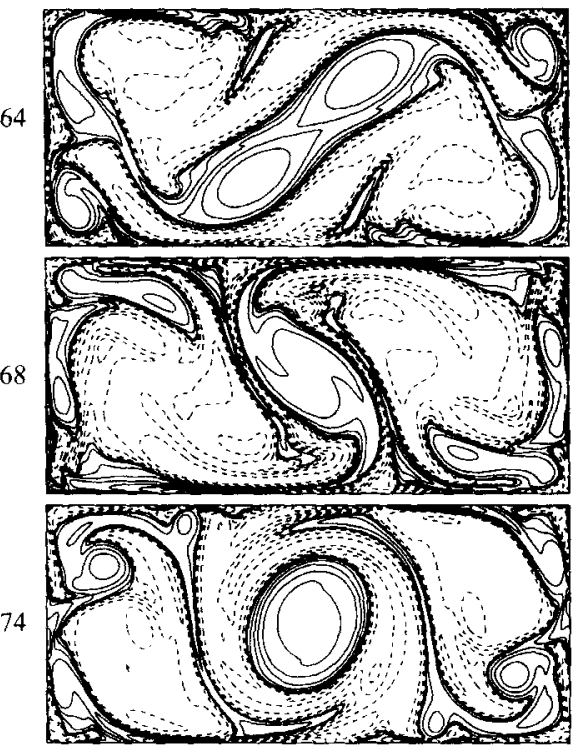

(b)
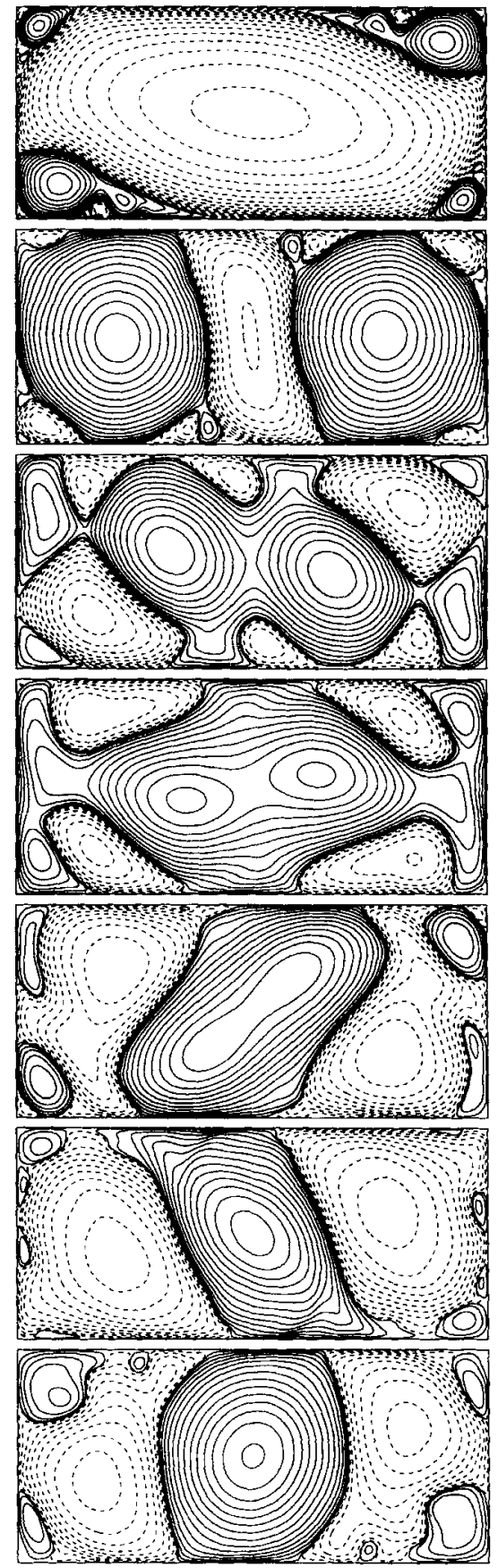

Figure 7. Contours of $\zeta(a)$ and $\psi(b)$ at the times as indicated for $R e=10^{4}$ and $\delta_{h}=2.20$ calculated a grid of size $301 \times 661$ with $\delta t=1.25 \times 10^{-3}$. Contour levels are as determined in figure 6. 
the cell patterns are $(+-+)$ with centres that reside on an axis at some angle $\theta$ to the long axis of the container. Figure $2(b)(t=95)$ shows that in the merger case, the cell patterns are $(-+-)$ with centres that reside on an axis at $-\theta$. We point out this observation recalling that the only solution to the unsteady problem as $t \rightarrow \infty$ is that of solid-body rotation, with $\psi=0$ and $\zeta=0$. Figure 8 shows this horizontal symmetry of the non-merger and merger patterns. It consists of photographs of the pathlines (near spin-up) of non-merger events (figure 8i) and merger events (figure 8ii) as $\delta_{h}$ is varied from 3 to 2. Variations in $\theta$ with $\delta_{h}$ remain small for $\delta_{h}>2.5$. For $\delta_{h}<2.5$ counter-rotating vortices in the trailing corners become significant.

\subsection{Critical Reynolds number for cyclone merger}

Experiments were also conducted in which the Reynolds number, $R e$, and the horizontal and vertical aspect ratios, $\delta_{h}$ and $\delta_{v}$, were varied to determine parameter regimes for the occurrence of the two patterns discussed in $\$ 6.1$. The primary result is that vortex merger occurs at some critical Reynolds number, dependent upon $\delta_{h}$ and $\delta_{v}$. We denote $R e_{c}$ as the lowest $R e$, for a given $\delta_{h}$ and $\delta_{v}$, at which merger occurs. Nevertheless, in some experiments with $R e>R e_{c}$ the cyclones did not merge.

Below some $R e_{c}$ the cyclonic vortices initiated in the leading corners filled their respective approximate thirds of the container, as in figure 2(a). Near $\operatorname{Re}_{c}$ the cyclones travelled to the centre of the container and merged as shown in figure $2(b)$. Figure 9 shows a parameter space of experimental results for $\delta_{v}=0.79$, for which the observed flow patterns are classified (see the discussion below). The main result is that $R e_{c}$ increases with increasing $\delta_{h}$. We note that computations give a significantly lower $R e_{c}$ than observed in the experiments. Since $R e_{c}$ is captured only qualitatively by the numerics, further quantitative comparisons, such as a numerically obtained parameter space corresponding to figure 9 , were not attempted.

The parameter space mapped out in figure 9 shows that for Reynolds numbers above $R e_{c}$, the pattern of flow evolution was complicated. For $\delta_{h} \approx 2$, the flow pattern was fairly ordered and exhibited merger as in figure $2(b)$. For $\delta_{h} \approx 3$, the flow exhibited a number of different patterns. The various observed pathlines above $R e_{c}$ are classified as patterns in which the vorticity at the centre was as follows.

(a) (•) Anticyclonic from a non-merger case, as shown in figure 2(a).

(b) (o) Cyclonic from a merger event, as shown in figure $2(b)$.

(c) (a) Cyclonic from either a merger event, unlike that shown in figure $2(b)$, or from a situation in which the horizontal symmetry of the rectangular container was broken resulting in pathlines that were complicated and disordered. Such an experiment is shown in figure $10(a)$. Figure $10(b)$ shows a similar flow structure for vorticity from the computations, which were also conducted at $R e>R e_{c}$. In both the experiment and computation of figure 10 the $\pi$-rotational symmetry of the system described in $\S \S 5$ and 6.4 was broken leaving one of many anomalous patterns that may arise at $R e>R e_{c}$. This anomalous pattern occurring in the numerics shows that Ekman pumping is not required to break this symmetry.

$(d)(\diamond)$ Cyclonic from a merger event unlike that shown in figure $2(b)$, but resulting in pathlines that showed a clean 3-cell pattern like that in figure $2(b)$.

$(e)(\triangle)$ Neither cyclonic or anticyclonic; here the cyclonic cells travelled toward the centre of the container, touched and remained stable, joined at the centre. The instantaneous stream function had a saddle point rather than a centre at the container centre. This configuration was stable once it was set up, but was not always reproducible for the parameters shown in figure 9 . In some experiments the small anticyclones at the top and bottom of the container centre would slowly shear fluid 
(i)

(a)

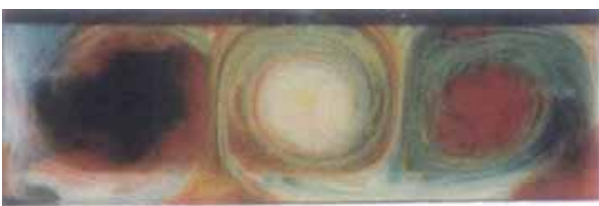

(b)

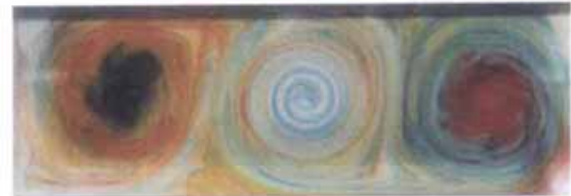

(c)

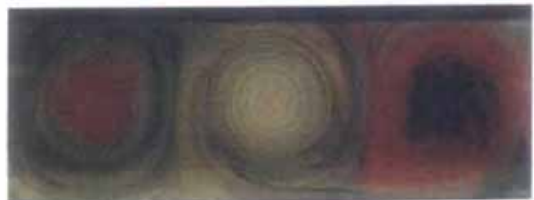

(d)

(e)

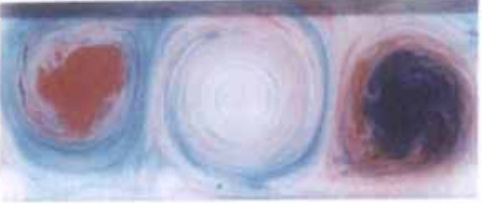

$(f)$
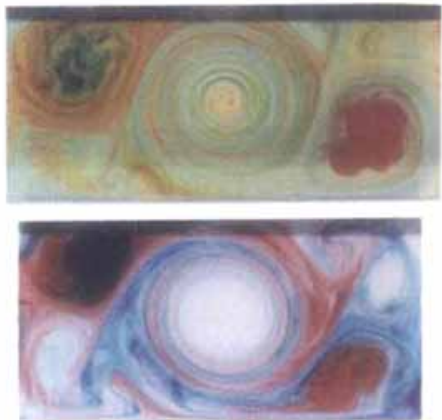

(ii)
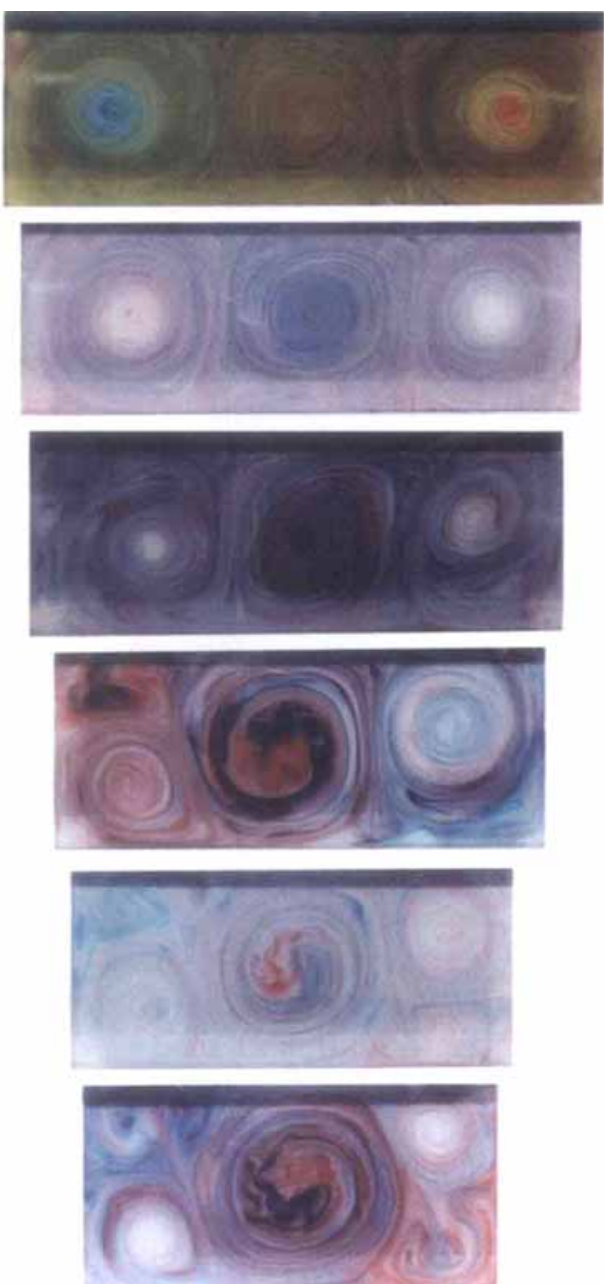

FiguRE 8 . Overhead photographs of the dye-line patterns near spin-up for non-merger experiments (i) and merger experiments (ii) in which $\delta_{v}=0.79$ and $L=12.7 \mathrm{~cm}$, where (a) $\delta_{\mathrm{h}}=3.0$, (i) $R e=5.00 \times 10^{3}$, (ii) $R e=2.62 \times 10^{4}$; (b) $\delta_{h}=2.8$, (i) $R e=6.55 \times 10^{3}$, (ii) $R e=2.61 \times 10^{4}$; (c) $\delta_{h}=2.6$, (i) $R e=6.55 \times 10^{3}$, (ii) $R e=2.46 \times 10^{4}$; (d) $\delta_{h}=2.4$, (i) $R e=6.55 \times 10^{3}$, (ii) $R e=1.30 \times 10^{4}$; (e) $\delta_{h}=2.2$, (i) $R e=6.55 \times 10^{3}$, (ii) $R e=1.46 \times 10^{4} ;$ (f) $\delta_{h}=2.0$, (i) $\operatorname{Re}=6.55 \times 10^{3}$, (ii) $R e=1.47 \times 10^{4}$

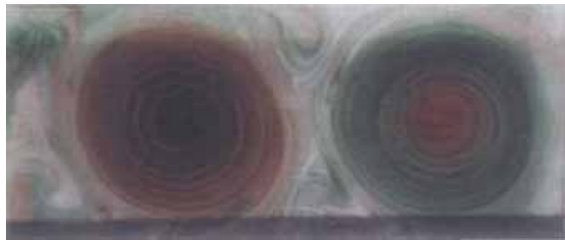

FigurE 11. An example of the two-celled pattern with a saddle point in the centre of the container. Here, $\delta_{t}=0.79, \delta_{h}=2.4$, and $R e=2.13 \times 10^{4}$. 


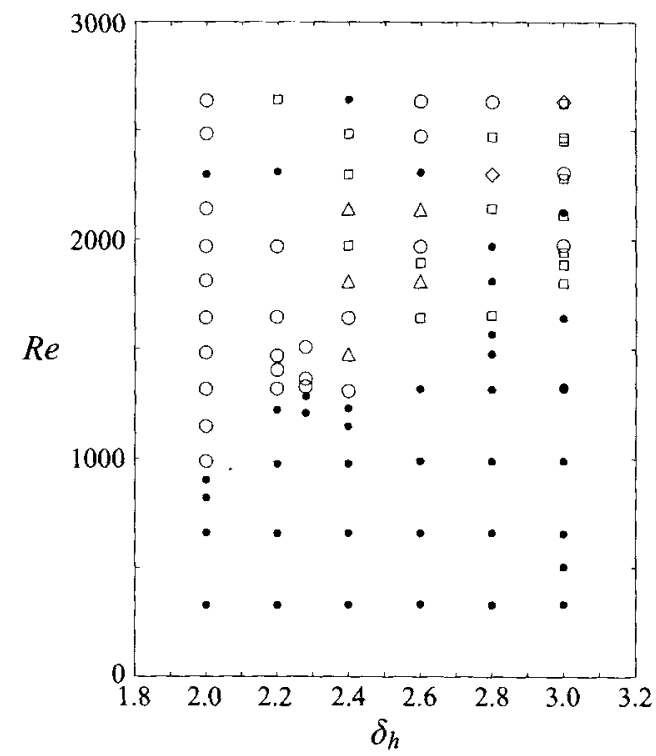

FIGURE 9. Classification of observed patterns of dye-line evolution as a function of $R e$ and $\delta_{h}$ for $\delta_{v}=0.79$. Patterns are classified as $\bullet(+-+)$, as in figure $2(a) ; \circ(-+-)$, as in figure $2(b) ; \square$, one with a cyclonic centre resulting from symmetry breaking that may or may not have involved cyclone-merger; $a$, merger that was not like that in figure $2(b)$, but resulted in the pattern shown for $t=95$ in figure $2(b)$; and $\triangle$, a two-celled pattern with a saddle point in the centre of the container, rather than a centre.

from the cyclones until the two anticyclones would join, separate the cyclones and result in the formation of the $(+-+)$ pattern shown in figure $2(a)$. A stable example is shown in figure 11 (see page 125).

We note that all of the results in figure 9 were obtained from a single container, container 1 in table 1 , that used spacers at the endwalls to change $\delta_{h}$. Results for $R e<R e_{c}$ were reproducible. The complicated flow patterns for $R e>R e_{c}$ were not necessarily reproducible; nevertheless, the qualitative behaviour of merger and symmetry breaking was reproducible.

\subsection{Effects of finite depth}

Figure 12 shows the dependence of the critical Reynolds number on the vertical aspect ratio for a fixed horizontal aspect ratio. The main result is that $R e_{c}$ does not vary monotonically with increasing $\delta_{v}$. Instead, cyclone evolution is markedly different for $\delta_{v}$ above and below 1 . For $\delta_{v}<1, R e_{c}$ seems to decrease rapidly with increasing $\delta_{v}$. For quite small $\delta_{v}$, the fluid spun up before the patterns in figure 2 were fully realized. For example, the merger events for $\delta_{v}=0.20$ only had time to evolve to a state between the third and fourth photo of figure $2(b)$. This result is in accordance with the previous result that Ekman pumping becomes an important mechanism in the spin-up process when $T_{E}$ is less than approximately $10 / \Omega$.

For $\delta_{v}=0.8$ the critical Reynolds number for merger was quite small. This was true in two different boxes with similar $\delta_{h}$. For $\delta_{v}=0.95$, the experimental results were unusual. The first merger event was at a low Re, but non-merger events proliferated for further increases in $R e$. For some values of the external parameters, both merger and non-merger events were observed with $\delta_{v}=0.95$. These merger and non-merger cases occurring in the $\delta_{v}=0.95$ container for the same parameters 
(a)

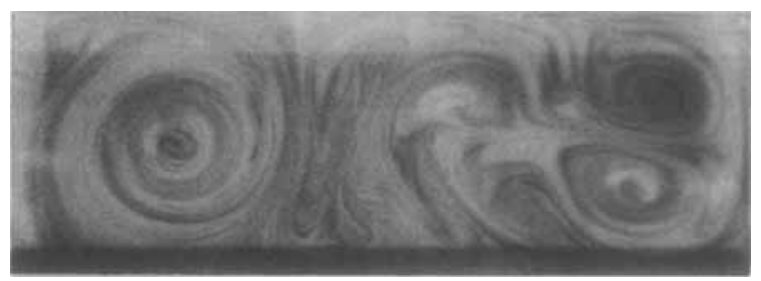

(b)

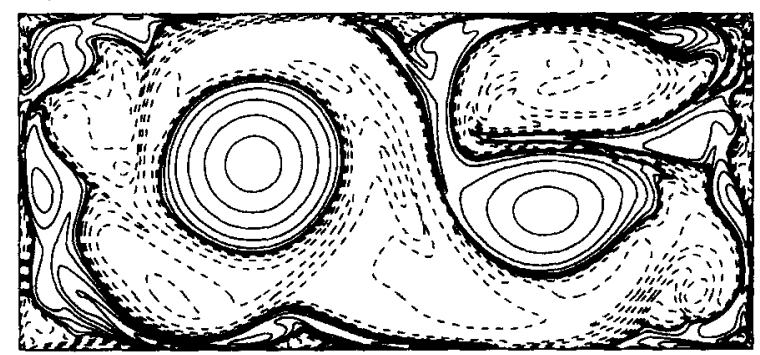

Figure 10. (a) Photograph of flow near spin-up with $R e=1.64 \times 10^{4}, \delta_{h}=2.8, \delta_{1}=0.79$ and $L=12.7 \mathrm{~cm}$. (b) Contours of computed $\zeta$ of a flow at $t=60$ with $R e=1.29 \times 10^{4}$ and $\delta_{h}=2.20$.

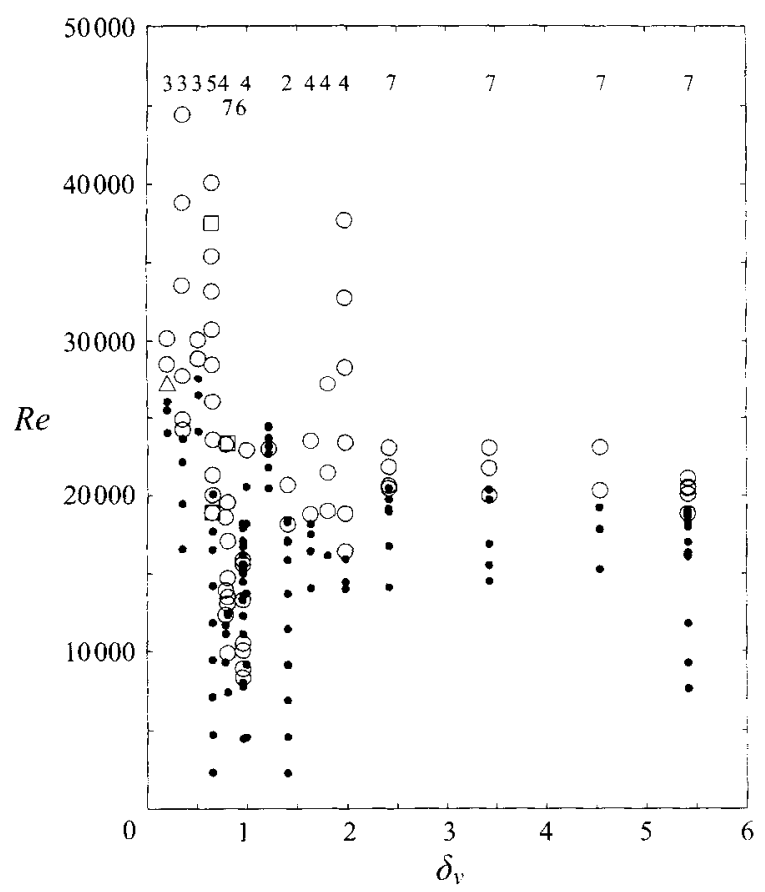

Figure 12. Experimentally determined patterns of dye-line evolution as a function of $R e$ and $\delta_{v}$ for $\delta_{h}=2.28$. Patterns are classified as $\bullet(+-+)$, as in figure $2(a) ; \circ(-+-)$, as in figure $2(b)$; $\square$, with a cyclonic centre resulting from symmetry breaking that may or may not have involved cyclone-merger; and $\triangle$, a two-celled pattern with a saddle point in the centre of the container. The numbers above each column of data identify the container used for the experiments at that $\delta_{v}$ (see table 1). 
are an example of the reflectional symmetry described by (5.1) and (5.2). However, the existence of multiple states at low $R e$ for fixed parameters was not observed in experiments with other containers, for which the results depicted in figure 12 (as in figure 9) were reproducible for $R e<R e_{c}$. Also as in figure 9, for experiments in the other containers with $R e>R e_{c}$, the details of the complicated patterns were not necessarily reproducible, but the basic phenomena of symmetry breaking and merger were. We note that the container with $\delta_{v}=0.95$ was made of the same materials as the other containers and was fastened in the same fashion. The results in figure 12 were obtained in six containers, indicated at the top of each column of data, corresponding to the labels in table 1.

In experiments for which $\delta_{v}=1.2$ the critical Reynolds number was quite large. One merger event was observed, but was not reproducible. In general, the cyclones did not merge for Reynolds numbers obtainable within the mechanical constraints of the experiment. In experiments for which $\delta_{v}>1.2, R e_{c} \approx 2 \times 10^{4}$ and changed little with increasing vertical aspect ratio. The small change in critical Reynolds number with increasing depth indicates that $\delta_{v}$, would have to be increased by about an order of magnitude before achieving the two-dimensional limit of $\approx 6.5 \times 10^{3}$ that was obtained in the two-dimensional computations. We also note that the observed times at which merger occurred did not depend significantly on $\delta_{v}$.

All of the experiments discussed above were conducted in containers with a rigid lid. When the experiments were conducted with a free surface, the behaviour of the flow was qualitatively the same as described above; however, the critical Reynolds number for merger was about $40 \%$ less, and the times for merger were less, than in experiments with a rigid lid. Since the surface deformation is small (Froude number, $\left.\Omega^{2} L^{2} / g H \sim O\left(10^{-2}\right)\right)$, these free-surface experiments essentially correspond to rigid-lid experiments with twice the depth.

\subsection{Variable initial conditions}

All of the experiments discussed above used the impulsive start for an initial condition. However, in some cases the results did change if the container was varied linearly from zero rotation to the final rotation rate over some finite time period. For example, for a container with $\delta_{h}=2.28, \delta_{v}=0.95$ and $R e=1.22 \times 10^{4}$, the cyclonic vortices did not merge when the start-up was impulsive. However, merger did occur when the change in rotation rate was varied linearly over $0.1 \mathrm{~s}$, or 0.018 of a rotation period. For this same container with $R e=1.33 \times 10^{4}$, the cyclones did not merge with an impulsive start-up or for a linear ramp time of $0.1 \mathrm{~s}$, but did merge for a ramp time of $1 \mathrm{~s}$, or 0.19 of a rotation period. For $R e=1.56 \times 10^{4}$ the cyclones did not merge with an impulsive start-up or for linear ramps of 1,2 or $5 \mathrm{~s}$, the last of which is 1.12 of a rotation period.

\section{Tracks of the cyclonic cells}

The most visually striking aspect of this flow is the early formation of cyclonic cells at the corners following the impulsive start-up. It is tempting to try and describe their subsequent evolution in terms of the dynamics of isolated vorticies; however, these cells are not isolated vorticies, and, while many aspects of their dynamics are very similar, they do not behave as such.

Although the vortex merger shown in figure $2(b)$ is quite reminiscent of other types of merger events, e.g. the merger of two identical isolated patches of uniform vorticity in an irrotational background (Dritschel 1986), two-dimensional vortex merger in 


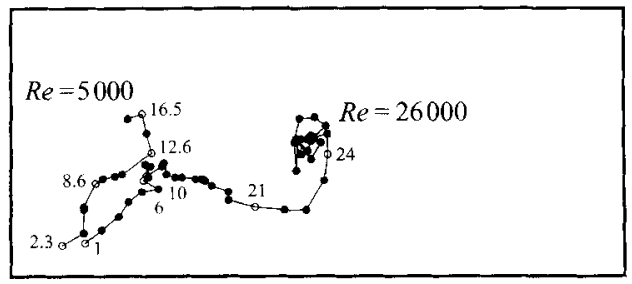

FIGURE 13. Measured tracks of cyclone centres for $\delta_{h}=2.28$ and $R e$ as indicated. The dye-line patterns near spin-up are characterized by the non-merger pattern of figure $2(a)$ for the $R e=5 \times 10^{3}$ track, and the merger pattern of figure $3(b)$ for the $R e=2.6 \times 10^{4}$ track. Times are also indicated on the figure.

isolation for very large Reynolds number flow with 'hyperviscosity' to suppress smallscale motions (Melander, Zabusky \& McWilliams 1988), and the merger of two identical vortices in a uniform strain field in an unbounded domain, over a range of Reynolds numbers (Buntine \& Pullin 1989), the details are different due to the effects of the sidewalls. The merger event in figure $2(b)$ also has many features in common with the photographs of the experiment conducted by Griffiths \& Hopfinger (1991), where two identical cyclones undergo merger. Their experiments were designed to minimize the effects of the sidewall; the cyclones were introduced into an environment with uniform background vorticity, and were conducted with a free surface. The cyclones in our experiment are not evolving in isolation; the vorticity produced by the acceleration of the sidewalls is of both signs. The positive vorticity primarily rolls up to produce the cyclones, and the negative vorticity is primarily wrapped around the cyclones. Even in the low $R e=2.36 \times 10^{3}$ case (figure $2 a$ ), there is evidence of secondary boundary layer separation feeding anticyclonic vorticity into the interior. The flow visualizations do not give a very clear picture of this, and we appeal to the two-dimensional numerical model of the primary interior flow for some indication of the processes involved.

In spite of the associated problems (see $\S 3$ ) it is instructive to attempt to identify the cyclone centres and follow them in time. Figure 13 presents non-merger and merger cases from the experiments; figure 14 shows cyclone tracks from computations. From the numerical model, the centres of the instantaneous streamlines were tracked, rather than the maximum of the cyclonic vorticity. The two give slightly different tracks, and the choice was made because the centres of the instantaneous streamlines are not only local maxima but, in their respective halves of the container, they are also global maxima, whereas the vorticity maxima at different times occur in the boundary layers or the separated shear layers or in the cyclonic cells, and automated centre detection is not straightforward. The dots on the tracks in figure 14 are equally spaced in time (every 4 time units) in order to give some indication of the speed of the cyclones at the various stages of the evolution.

The tracks for $R e<10^{4}$ are virtually indistinguishable for $t<20$. In essence, this demonstrates the inertia of the cells causing them to remain stationary in the laboratory frame. For low $R e$, these cells remain monopolar for all time, viscous forces aiding to keep them smooth and circular, and their tracks smooth. They eventually reach locations $(1.47,-0.18)$ and $(-1.47,0.18)$, which we shall simply refer to as the $(+-+)$ locations. In experiments with the rigid lid, the final location of the cyclones is essentially independent of $R e$, in accordance with the numerical results. However, 

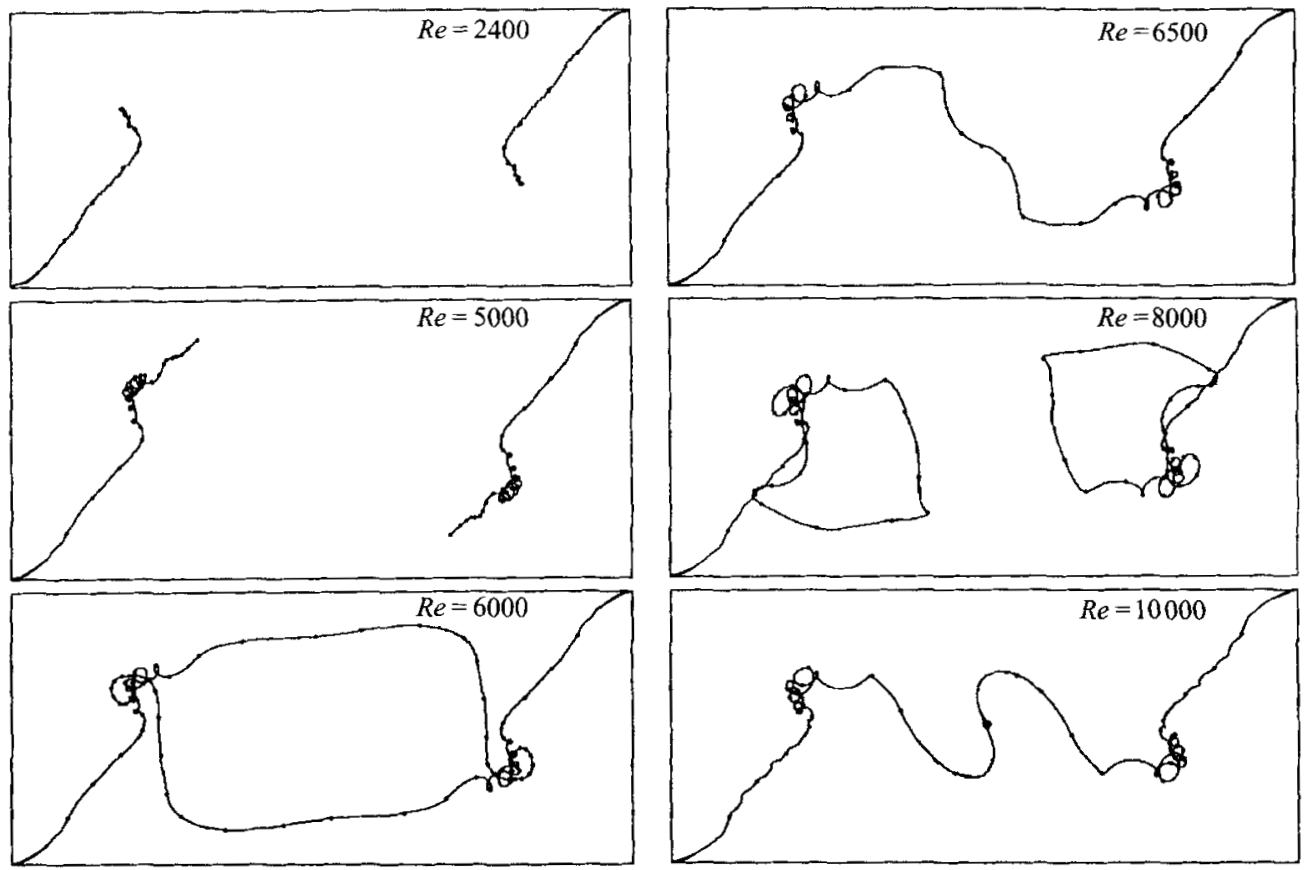

FigURE 14. Tracks of cyclone centres for $\delta_{h}=2.28$ and $R e$ as indicated. The dots on the tracks are spaced four time units apart. The tracks are of the centres of the instantaneous stream function corresponding to the cyclonic cells, calculated on the $(301 \times 685)$ grid, except for the $R e=2.36 \times 10^{3}$ case which used a $(151 \times 343)$ grid, with $\delta t=1.25 \times 10^{-3}$.

Stewart (1993) reported that in experiments with a free surface, the cyclones tend to migrate closer to the centre as $R e$ is increased.

At higher $R e$ (e.g. $R e=5 \times 10^{3}$ as shown in figure 14), the cyclone tracks take on a trochoidal motion as they approach the $(+-+)$ locations. This trochoidal motion of the cyclone centres is also observed in the present experiments with a rigid lid and in the free-surface experiments of Stewart (1993). At these $R e$, the numerics suggest that the trochoidal motion is induced by the feeding of anticyclonic vorticity from the sidewall layer at the secondary separation point. This anticyclonic vorticity tends to wrap around the cyclonic cell and at times aggregates to form small anticyclonic cells, thus producing a multi-polar cell complex. These satellite anticyclonic cells rotate about the cyclone in the cyclonic direction. They are short lived, surviving only on the order of one rotation time around the cyclone before they are annihilated by viscous diffusion, but new anticyclonic cells are fed in from the sidewall layers. Their presence effectively steers the cyclone off the smooth track, resulting in the observed trochoidal motions. At later times $(t>90)$, the cyclone centres drift away from the $(+-+)$ locations. For $R e=5 \times 10^{3}$, the cyclones have spun down considerably by $t=90$ and although centres of $\psi$ are still identifiable, they no longer constitute centres of vortical cells. For $R e=6 \times 10^{3}$ however, the cyclones' peak $\zeta$ is greater than 2 at late times, and they track along the top and bottom sidewalls. The cyclones eventually settle in the $(+-+)$ locations, but diagonally opposite to the locations each cyclone initially tracked to. The panels in figures 13 and 14 covering a large range of $R e$ and dynamics demonstrate that in all cases the cyclones initially track towards the $(+-+)$ locations. 
For $R e=10^{4}$ (figure 14), the trochoidal motion begins at very early times (however, the loops are not as evident in the figure as there is a large linear component to their motion). In this case, the cyclonic cell has a thin, intense layer of anticyclonic vorticity wrapped around it from a very early time. As depicted in figure 7 at $t=18$, which shows a very similar flow corresponding to $R e=10^{4}$ and $\delta_{h}=2.2$, there is also a thin intense layer of cyclonic vorticity wrapping around the thin layer of anticyclonic vorticity, and by this early time, the cyclonic cell already has some satellite vorticies circling it, causing the trochoidal motion. These two shear layers originate from the boundary layer on the wall at $y=-0.5$. There is another shear layer wrapping around the cyclonic cell originating from the boundary layer on the wall at $x=-W / 2 L$, and also a shear layer wrapping around from the wall at $y=0.5$.

At $t=60$, the shear layers of anticyclonic vorticity from the walls at $y=0.5$ and $x=-W / 2 L$ have combined and rolled up to produce an anticyclonic cell which links up with the cyclonic cell to produce a skewed dipolar cell, with the cyclonic part being dominant. The dipole on the left begins to migrate to the right and the one on the right migrates to the left. By $t=78$ (merger occurs earlier for the lower- $\delta_{h}$ case shown in figure 7) the cyclonic parts of the two dipoles undergo merger, as depicted in figure 7, to form a cyclonic cell in the centre of the container. The anticyclonic parts of the cyclones eventually settle down to produce the anticyclones flanking the cyclone in the $(-+-)$ configuration. Notice however, that at $t=74$, the configuration is not a simple $(-+-)$ formation: there are small, weak cyclonic cells near the trailing corners as well. The flow visualizations (e.g. figure $2 b$ at $t=95$ ) also show dye-lines which are suggestive of the presence of weak cyclones near the trailing corners following a merger event.

The criterion for the inviscid merger of two identical, isolated patches of uniform vorticity (Zabusky, Hughes \& Roberts 1979), based on the ratio of their separation to their radius is not appropriate here, where the cyclones are not isolated. Further, experiments show that, in fact, it does not hold here. For example, figure 11 shows an experiment in which two cyclones actually touched in a stable configuration without merging. The cyclonic cells initially always tend to migrate towards the $(+-+)$ locations, regardless of whether they subsequently merge or not. Their radius is primarily determined by $L, \delta_{h}$, and to some extent by $R e$. Cyclones tend to have smaller radii for larger $R e$. Yet, we find that merger occurs for larger $R e$. This is not to suggest that the merger events observed here are viscous processes, but rather that the conditions required for merger are $R e$ dependent. Primarily, we feel, $R e$ determines the intensity of the cyclonic cells following the roll-up of the separated sidewall layers and how fast their vorticity is diffused compared to their convection speed.

Our merger events share some similarities with the scenario presented by Melander et al. (1988). Their criterion, based on the inviscid moment-model equations and verified by numerical computations of the Euler equations, hinges on the existence of a stable state. The possibility of merger depends on whether the angular impulse

$$
\sigma=\iint \zeta(x, y)\left(x^{2}+y^{2}\right) \mathrm{d} x \mathrm{~d} y
$$

of the two symmetric isolated vortices is above or below some critical value, $\sigma_{c}$, determining the existence of a steady co-rotating state. For $\sigma<\sigma_{c}$, there is no steady co-rotating state, so merger will occur for all initial conditions. Whereas for $\sigma>\sigma_{c}$, both stable and unstable states exist, and merger may or may not occur, depending on initial conditions. Our observation that the cyclonic cells in the two-dimensional 
model, for all $R e$ considered, initially tend towards the $(+-+)$ locations is suggestive of an underlying attractor. However, the flow is continuously evolving (spinning up) and there are no conserved quantities, so the concept of a stable state is not clear. Further, the effects of sidewalls and the distribution of other vortical flow are not accounted for in the Melander et al. (1988) scenario. Another important aspect of their scenario is the production of a tangle of nearly concentric vortex filaments following the merger. While both the experimental dye-lines (figure $2 b$ ) and the computations from the two-dimensional model (figure 7) give evidence of thin vortical strands wrapped around the central cyclone following merger, it is not clear to what extent these are composed of fluid expelled from the cyclones during the merger or whether they are vortical fluid extracted from the sidewall layers following separation induced by the interior flow.

The case of $R e=8 \times 10^{3}$ (figures 14 and 15) also shows evidence of the influence of the sidewalls on the merger. In this case, the two cyclones begin to merge as in the other merger cases; however, part way into the merger, as the two cyclones begin to rotate about their common centre cyclonically and become elongated, they come into contact with the sidewall. At this point, rather than continue with the merger, they each travel along the sidewalls and eventually reside in the $(+-+)$ locations. This result is also observed in the experiments for which $\delta_{v}>4$. It was not observed in experiments in shallower containers, where presumably the vertical flow that tends to enhance merger dominates the sidewall effects that might act to inhibit it.

\section{Summary}

Investigation of the impulsive spin-up of a fluid from rest in non-axisymmetric containers has shown that the spin-up process can be dominated by a process not present in axisymmetric containers (at least while the flow in the axisymmetric containers retains azimuthal symmetry). This additional process of transporting angular momentum into the interior is provided by the separation of the sidewall boundary layers, formed following the impulsive start, and their roll-up into cyclonic cells in on the order of one rotation time $(1 / \Omega)$ of the system. Horizontal viscous diffusion and Ekman pumping also provide mechanisms for spin-up, but for the parameter ranges considered in this study, these are secondary processes. Specifically, for spin-up in rectangular containers of depth $H$ of the same order or larger than the short horizontal length of the container $L$, if $\left(H^{2} / \nu \Omega\right)^{1 / 2}$ is greater than approximately $10 / \Omega$, then the advection of angular momentum into the interior by the cyclonic cells occurs on a faster time scale than Ekman pumping. Numerical computations of the two-dimensional Navier-Stokes equations qualitatively capture much of the dynamics of the flow for these cases.

For spin-up at low $\operatorname{Re}\left(<R e_{c}\right)$, the experiments and the numerical computations of the limiting case of $H \rightarrow \infty$ show that the cyclones, locally, rotate faster than the container, and hence the spin-up of the entire system is essentially a local spin-down of the cyclonic cell regions, coupled with a spin-up of the initially stationary interior into an anticyclonic cell by the flanking cyclones via viscous shear. For spin-up with $R e \geqslant R e_{c}$, the cyclones track into the interior and may undergo merger, forming a system with one cyclonic cell in the centre. This resultant cyclonic cell then spins down locally as the system approaches solid-body rotation. The cyclone-cyclone merger events appear to be very similar to mergers of isolated vortices; however, both the experiments and the numerics show that the close proximity of the sidewalls and the associated distribution of negative (anticyclonic) relative vorticity provide 
(a)
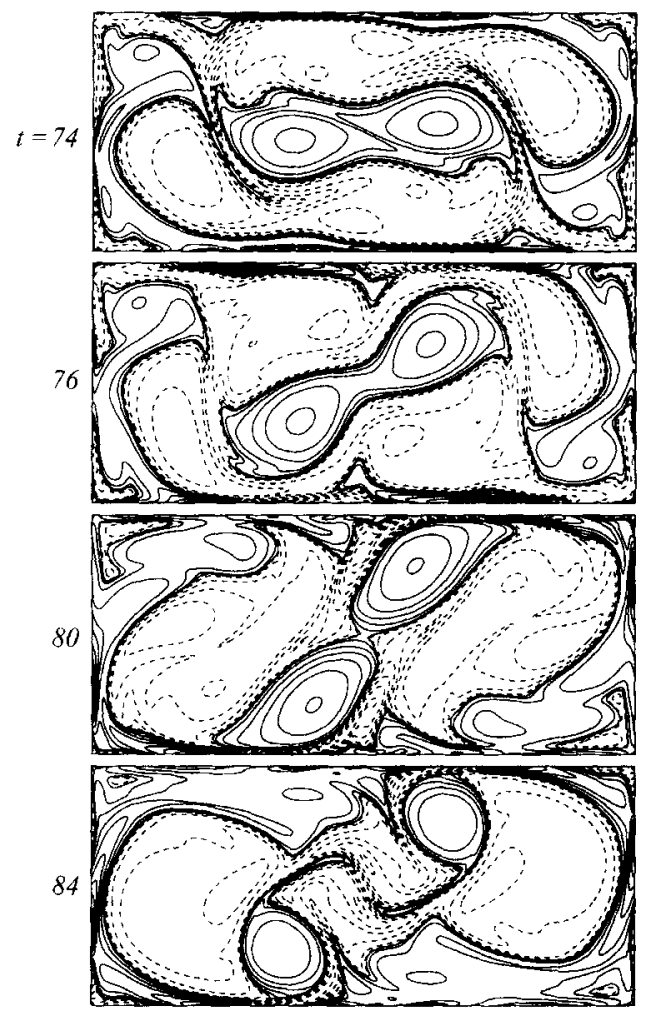

(b)
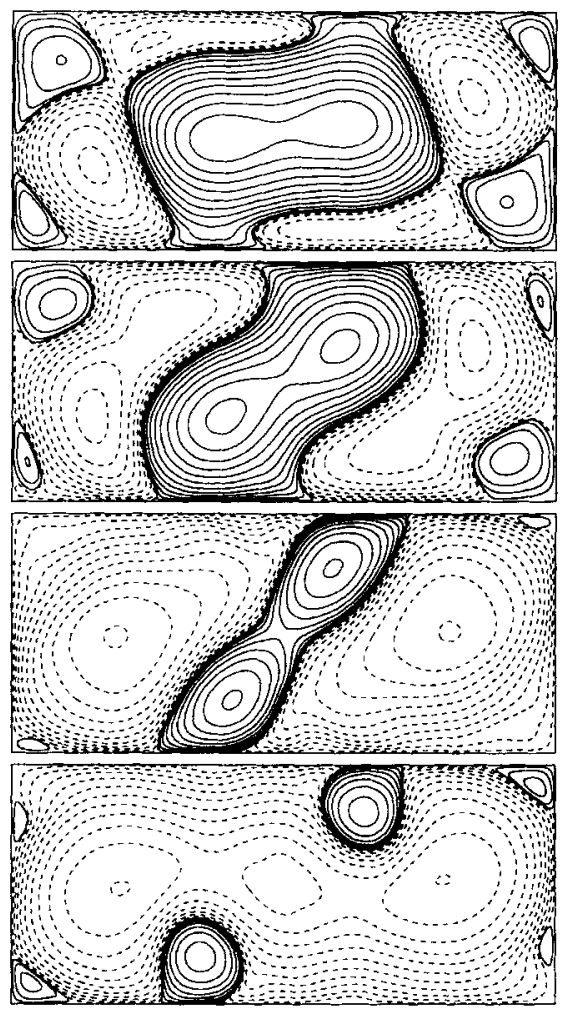

FIGURE 15. Contours of $\zeta(a)$ and $\psi(b)$ at times as indicated for $R e=8 \times 10^{3}$ and $\delta_{h}=2.28$ calculated with a grid of size $301 \times 685$ with $\delta t=1.25 \times 10^{-3}$. Contour levels are as determined in figure 6.

further complications for the mergers. The critical Reynolds number for merger increases with increasing horizontal aspect ratio at a fixed vertical aspect ratio. It varies non-monotonically with increasing vertical aspect ratio at a fixed horizontal aspect ratio. In particular, $R e_{c}$ decreases sharply with increasing $\delta_{v}$ for $\delta_{v}<1$, then jumps at $\delta_{v}=1$, and decreases at a lower rate for further increases in $\delta_{v}$.

We are grateful for helpful discussions with Joe Hammack, John Hart, Jie Shen, Simon Tavener and Patrick Weidman, and to Bob Geist, who built the experimental apparatus. This work was funded by the National Science Foundation under DMS9257456, the David and Lucile Packard Foundation, the USAF, and the Pennsylvania State University. Many of the computational resources were provided by the Center for Academic Computation of the Pennsylvania State University.

\section{REFERENCES}

Benton, E. R. \& Clark, A. 1974 Spin-up. Ann. Rev. Fluid Mech. 6, 257-280.

Buntine, J. D. \& Pullin, D. I. 1989 Merger and cancellation of strained vortices. J. Fluid Mech. 205, 263-295.

DRITSCHEL, D. G. 1986 The nonlinear evolution of rotating configurations of uniform vorticity. J. Fluid Mech. 172, 157--182. 
Griffiths, R. W. \& Hopfinger, E. J. 1991 Coalescing of geostrophic vortices. J. Fluid Mech. 178, 73-97.

HeIJST, G. J.F. vaN 1989 Spin-up phenomena in non-axisymmetric containers. J. Fluid Mech. 206, $171-191$.

Heijst, G. J. F. van, Davies, P. A. \& Davis, R. G. 1990 Spin-up in a rectangular container. Phys. Fluids A 2, 150-159.

JeONG, J. \& Hussain, F. 1995 On the identification of a vortex. J. Fluid Mech. 285, 69-94.

Kitchens, C. W. 1980 Navier-Stokes solutions for spin-up in a filled cylinder. AIAA J. 18, 929-934.

KONIJNENBERG, J. A. VAN DE 1995 Spin-up in non-axisymmetric containers. $\mathrm{PhD}$ thesis, Fluid Dynamics Laboratory, Department of Physics, Eindhoven University of Technology, The Netherlands.

Konimnenberg, J. A. van de, Andersson, H. I., Billdal, J. T. \& Heisst, G. J. F. van 1994 Spin-up in a rectangular tank with low angular velocity. Phys. Fluids 6, 1168-1176.

Melander, M.V., Zabusky, N.J.\& McWilliams, J.C. 1988 Symmetric vortex merger in two dimensions: causes and conditions. J. Fluid Mech. 195, 303-340.

Pedlosky, J. 1984 Geophysical Fluid Dynamics. Springer.

Stewart, D. L. 1993 Spin-up in a rectangular cylinder. Master of Arts Thesis, Department of Mathematics, The Pennsylvania State University.

SuH, Y. K. 1994 Numerical study on two-dimensional spin-up in a rectangle. Phys. Fluids 6, 23332344.

SwEET, R. A. 1974 A generalized cyclic reduction algorithm. SIAM J.Numer. Anal. 10, 506-592.

WEAST, R. C. 1976 (ed.) Handbook of Chemistry and Physics, 57th Edn. CRC Press.

WEIDMAN, P.D. $1976 a$ On the spin-up and spin-down of a rotating fluid. Part 1. Extending the Wedemeyer model. J. Fluid Mech. 77, 658-708.

WeIDMAN, P. D. $1976 b$ On the spin-up and spin-down of a rotating fluid. Part 2. Measurements and stability. J. Fluid Mech. 77, 709-735.

WILLIAMS, G. P. 1967 Thermal convection in a rotating fluid annulus: Part 1. The basic axisymmetric flow. J. Atmos. Sci. 24, 144-161.

Zabusky, N.J., Hughes, M. H.\& RoberTs, K.V. 1979 Contour dynamics for the Euler equations in two dimensions. J. Comput. Phys. 30, 96-106. 